\title{
Quantification and analysis of the nonlinear effects in spectral broadening through solid medium of femtosecond pulses by neural network
}

\author{
Yitan Gao $\odot,{ }^{1,2}$ Xianzhi Wang, ${ }^{1,2}$ Xiaoxian Zhu, ${ }^{1,2}$ Kun Zhao $\odot,{ }^{1,3, *}$ Heyuan Liu, ${ }^{1,2}$ \\ Zhaohua Wang, ${ }^{1}$ Shaobo Fang, ${ }^{1,2,3}$ and Zhiyi Wei ${ }^{1,2,3, \dagger}$ \\ ${ }^{1}$ Beijing National Laboratory for Condensed Matter Physics, Institute of Physics, Chinese Academy of Sciences, Beijing 100190, China \\ ${ }^{2}$ Department of Physics, University of Chinese Academy of Sciences, Beijing 100049, China \\ ${ }^{3}$ Songshan Lake Materials Laboratory, Dongguan 523808, China
}

(Received 11 June 2021; revised 18 October 2021; accepted 7 December 2021; published 18 January 2022; corrected 27 June 2022)

\begin{abstract}
Supercontinuum generation has been widely applied in laser spectroscopy and few-cycle pulse generation. It is composed of complex and unmeasurable nonlinear optical effects, which influence the final broadened spectrum markedly. To describe and characterize the two key processes, the Kerr effect and ionization, we employ two nonlinear integrals, including the common B integral for the Kerr effect and a P integral for ionization or plasma effect. With these integrals, the contributions of Kerr and plasma effects in the supercontinuum generation are identified and determined quantitatively. Then we utilize machine learning to construct a multilayer perceptron neural network to obtain the solution of the propagation equation for a femtosecond pulse in a solid medium. We employ supervised and unsupervised training with both experimental and simulation data to train the neural network for a better accuracy of the calculation. The trained network can take the input and broadened spectra of the pulse to compute initial laser parameters and the B and P integrals instantly so that the contributions of Kerr and plasma effects in the supercontinuum generation may be quantified in real time, unveiling the nonlinearities behind the spectral evolution. This method provides a more accurate understanding of the physics of the entangled nonlinear optics effects and a faster and more convenient tool in the investigation of nonlinear optics.
\end{abstract}

DOI: 10.1103/PhysRevResearch.4.013035

\section{INTRODUCTION}

Attosecond optics is one of the most eminent frontiers of atomic, molecular, and optical physics, an energetic interdisciplinary research area combining strong-field physics and ultrafast optics [1]. The essence of attosecond optics is the generation of attosecond optical pulses through high-order harmonic generation $(\mathrm{HHG})$ and the examination of electronic motion on atomic scale with such pulses [2]. Transient spectroscopy with attosecond pulses ensures a compelling tool to enhance our understanding of ultrafast electronic processes in multibody systems, where the collective electronic motion and correlation mechanisms symbolize the core issues in both theoretical and experimental research [3].

However, one of the key issues in attosecond optics is that the photon flux in $\mathrm{HHG}$ is extremely low. Although extreme ultraviolet pulses $\sim 50$ as have been demonstrated [4,5], a typical attosecond pulse produced by millijoule-level femtosecond driving pulses contains only about $1 \mathrm{~nJ}$ energy [6,7], which is not enough to carry out autocorrelation, nonlinear optics, or multiphoton process experiments. To

\footnotetext{
*zhaokun@iphy.ac.cn

†zywei@iphy.ac.cn
}

Published by the American Physical Society under the terms of the Creative Commons Attribution 4.0 International license. Further distribution of this work must maintain attribution to the author(s) and the published article's title, journal citation, and DOI. characterize an attosecond pulse or perform an attosecond transient absorption experiment, a femtosecond laser pulse is usually required to engage atto-femto cross-correlation [5] or pump-probe [8] operations. The probability of two-photon ionization, the most basic and the lowest order nonlinear process, is determined by $P^{(2)}=\sigma^{(2)} \tau\left(I_{x} / E_{x}\right)^{2}$, where $\sigma^{(2)}$ is the two-photon ionization cross-section, while $\tau, I_{x}$, and $E_{x}$ are the pulse duration, intensity, and photon energy of the attosecond pulse. Due to the extremely small $\sigma^{(2)}$, the probability of two-photon ionization is terribly low, notably at low intensities. As a rule of thumb, we may state that the nonlinear process is observable when the probability of two-photon ionization equals that of one-photon ionization $P^{(1)}=\sigma^{(1)} \tau I_{x} / E_{x}$ with $\sigma^{(1)}$ as the single-photon ionization cross-section. Assuming $E_{x}=25 \mathrm{eV}$, just above the helium ionization potential at which either one- or two-photon ionization may occur, and taking $\sigma^{(1)} \approx 7.2 \times 10^{-18} \mathrm{~cm}^{2}[9,10]$ and $\sigma^{(2)} \approx 3 \times 10^{-52} \mathrm{~cm}^{4} \mathrm{~s}$ [11] for helium, an intensity of $I_{x} \approx 9.6 \times 10^{16} \mathrm{~W} / \mathrm{cm}^{2}$ is required to have $P^{(2)} \approx P^{(1)}$. For a pulse duration of 100 as and a beam diameter of $5 \mu \mathrm{m}$, a pulse energy of $1.9 \mu \mathrm{J}$ is required to reach such an intensity. Therefore, we may claim that $\sim 1 \mu \mathrm{J}$ pulse energy would be the lower limit to study nonlinear atto-optics.

Considering a conversion efficiency $\sim 10^{-5}$ to $10^{-7}$ for HHG and attosecond pulse generation $[6,12]$, one way to produce a $1 \mu \mathrm{J}$ attosecond pulse is to use a femtosecond pulse with $1 \mathrm{~J}$ energy. In attosecond pulse generation, few- or monocycle driving pulses would certainly be highly efficient since an isolated attosecond pulse is generated within only a half cycle of the driver [13]. The key component in an efficient 
high-energy attosecond source is, therefore, a high-energy femtosecond few-cycle driving laser.

Supercontinuum generation leading to coherent emissions in ultraviolet, visible, and infrared bands through self-phase modulation (SPM) $[14,15]$ of narrow-band driving pulses has been investigated since lasers were invented [16-18], realizing broadband radiation sources for a range of applications [19,20] including ultrafast spectroscopy [21,22], high-precision frequency metrology [23], optical coherence tomography [24], intrapulse difference frequency generation [25-27], seeding for an optical parametric amplifier [28] or optical parametric chirped-pulse amplifiers [29-31], highpower pulse compression [1,32-36], probing laser-plasma interactions [37], extreme nonlinear optics [38], short pulse synthesis [39-41] as well as HHG [42,43] and isolated attosecond pulse generation $[5,44,45]$. Coherent near-octave-, octave-, or multi-octave-spanning spectra, generated in gas [46-49], liquid [16,50-53], solid-state materials [32,33,52-62], or multiple thin plates (MTPs) of dielectrics [55,63-73] as well as in optical fiber [74-76], gas-filled hollow-core fiber (HCF) [39,41,77-87], gas-filled photonic crystal fiber [88], ZBLAN fiber [89], silicon photonic wire [90], silicon nitride waveguide [91], orientation-patterned gallium phosphide [92], cascade of different materials [93], or a nonlinear telescope [94], may be employed to produce few- to monocycle light pulses.

Nowadays, it is popular to compress multicycle femtosecond pulses with HCFs [77-87] or MTPs [64-73,95] plus chirped mirrors to generate few-cycle pulses. Since the beam exits a short slab of dielectrics before self-focusing starts to take effect, thin plates $[1,32,34,54]$ may be used to minimize catastrophic self-focusing [96,97], which causes optical breakdown and permanent damage in a solid medium [98-102]. To generate a continuum broad enough, SPM in multiple Kerr elements was proposed theoretically [103,104] and presented experimentally [55,63,64]. By dividing a bulk solid medium into separated thin pieces, the laser pulse is spectrally broadened via SPM in each thin plate, while the minimal beam cross-section (focal spot) produced by self-focusing is brought out of the high-density solid so that ionization and filamentation caused by self-focusing are greatly reduced $[64,68]$. Since generating few-cycle femtosecond pulses with MTPs was demonstrated [64], this scheme has been employed for different pulse energies, in different wavelength ranges [65-67,69-73,95], or in a cascade of compression setups [105-110]. Solid-state material also has the potential to generate high-power ultrafast laser pulses [1,34,111-113]. The advancement of high-power fewto monocycle laser sources [20] is critical for frontier applications such as high-energy attosecond pulse generation, nonlinear optics, ultrafast optics, and strong-field physics.

However, even with MTPs, conical emission [114,115], shown as the rings outside the center peak in the output beam, has been observed in experiments [64]. Conical emission appears when filamentation occurs, and experimental and numerical investigations have proved plasma generation to be one of the main factors resulting in such rainbow ring structures [116-118]. This indicates that serious ionization and plasma formation still exist even in thin plates. Surprisingly, self-compressed annular-shaped few-cycle pulses have been demonstrated in the rainbow rings formed by coni- cal emission in MTPs at $800 \mathrm{~nm}$ central wavelength [119], which makes conical emission a useful tool in few-cycle pulse generation.

Therefore, it is of extreme importance to understand ionization and plasma formation in the supercontinuum generation process [120] as femtosecond pulses propagate through a solid medium. Specifically, understanding the individual effect of SPM and ionization as well as the interplay between them is crucial to control the relative strength of these effects and may lead to a better characterization of the generated supercontinuum and a chance to produce shorter and stronger few-cycle pulses.

Unfortunately, the Kerr effect and ionization, as discussed above, are entangled with each other as short laser pulses propagate through the medium and cannot be measured or characterized individually in experiments. Traditionally, in nonlinear optics, a B integral [121] has been defined as $\mathrm{B}_{\mathrm{Kerr}}=\frac{2 \pi}{\lambda} \int_{0}^{z} \max \left(\Delta n_{\mathrm{kerr}}\right) d z$, where $z$ is the position in the propagation direction, $\Delta n_{\text {kerr }}$ the change of the refractive index of the medium induced by Kerr effect, and $\lambda$ the laser wavelength, representing the total nonlinear phase shift accumulated as the laser beam propagates through a medium, which is also an indicator of the strength or efficiency of the Kerr effect. In supercontinuum generation, the B integral also dictates how wide the spectrum is broadened [20]. On the contrary, there was no such quantity to describe ionization. However, the net change of the refractive index promoted by the laser field $n=n_{0}+\Delta n_{\text {kerr }}+\Delta n_{\text {plasma }}$ has contributions from both the Kerr effect and ionization (plasma formation), where $n_{0}$ is the linear refractive index. To describe the overall nonlinear effect as well as the interplay of Kerr and ionization effects, we define a $\mathrm{P}$ integral, like the $\mathrm{B}$ integral, to represent the phase change induced by plasma as well as the strength of ionization $\mathrm{P}_{\text {ion }}=\frac{2 \pi}{\lambda} \int_{0}^{z} \max \left(\Delta n_{\text {plasma }}\right) d z$. With these two parallel integrals, the two key processes in supercontinuum generation, the Kerr effect and ionization, may be described, characterized, and compared with each other.

In this paper, we investigate spectral broadening of femtosecond laser pulses in a solid medium numerically and experimentally. In the numerical simulations, we solve a three-dimensional (3D) nonlinear envelope equation (NEE) to predict the final output spectrum as well as calculate the $B$ and $\mathrm{P}$ integrals for the entire propagation through the medium. In the experiments, we measure the energies, spectra, and widths of the input and spectrally broadened pulses as well as the beam size on the medium to make comparison with the numerical results. However, solving the NEE numerically, especially in 3D, requires a large amount of computational power and is time consuming. At the same time, although solving the NEE produces values of all the output parameters in the numerical results, not all the parameters are measurable in experiments. The $\mathrm{B}$ and $\mathrm{P}$ integrals cannot be measured directly, while measuring the spectral phase of the output pulse is quite a challenging task. Among all the parameters of femtosecond pulses, only energy and spectra are readily accessible in experiments. Consequently, it becomes problematic to extract thorough physical insights from comparisons between experimental and numerical results.

Fortunately, this almost insurmountable problem seems to have found glimmers of hope with the evolution of machine 
learning. In the field of artificial intelligence, machine learning is one of the basic methods which makes decisions and predictions of real-world events by parsing and learning data through specific algorithms. It can establish a relationship between a series of seemingly causal data, and if the learning model is trained well, predictions of corresponding parameters may be obtained instantly. This method injects vigor into ultrafast laser field, and many works based on machine learning have been reported $[122,123]$. Among algorithms of machine learning, the artificial neural network, also termed as the multilayer perceptron (MLP), has been proved to be capable of approximating almost any continuous function universally [124], which means MLPs may be employed to fit the NEE. Unlike the traditional NEE solving process, the calculation of MLPs could be accelerated by parallel processing, which provides the possibility of finding the initial laser parameters from the broadened spectrum in real time.

Here, we apply a fully connected MLP neural network in the spectral broadening process to map the relationship between the broadened output spectrum and initial laser parameters as well as nonlinear parameters, including B and $\mathrm{P}$ integrals, as the laser pulse propagates through a piece of solid medium. Taking advantage of its efficient algorithm, the MLP network is capable of computing propagation parameters, only needing of the initial and final spectra. We realize reverse investigation on pulse propagation in the spectral broadening process as well as quantitative analysis of the key nonlinear processes in supercontinuum generation experimentally and numerically.

Due to its consistency in processing data and approximating continuous functions, a well-trained MLP network can combine simulated data with experimental results to realize the computation of parameters that cannot be measured experimentally. Through the network structure of the MLP, the measurable parameters are connected with the unmeasurable ones, which provides a rigorous mechanism for us to estimate the unmeasurable parameters of a physical process to gain deeper insights of the intermediate states not accessible before. This initiative will have a significant impact on the research and applications of spectral broadening, supercontinuum and few-cycle pulse generation, as well as nonlinear and ultrafast optics in general.

\section{PROPAGATION EQUATION}

To identify the relationships between spectra and initial laser parameters as well as the nonlinear effects that pulses experience during laser-matter interaction, we use the NEE to simulate the propagation process of a femtosecond pulse inside nonlinear material, which ensures that the numerical relationships obtained are firmly grounded in physical theory. The $2 \mathrm{D}+1$ NEE is written as

$$
\begin{aligned}
\partial_{z} U= & \frac{i}{2 k_{0}} T^{-1} \nabla_{\perp} U+i D U+i \frac{\omega_{0}}{c} n_{2} T|U|^{2} U \\
& -i \frac{k_{0}}{2 n_{0} \rho_{c}} T^{-1} \rho U-\frac{\beta^{(\kappa)}}{2}|U|^{2 \kappa-2} U-\frac{\sigma}{2} \rho U,
\end{aligned}
$$

where the optical processes such as dispersion, SPM, selfsteepening, multiphoton absorption and plasma formation are introduced. Here $U(r, z, t)$ is the electric field envelope, $k_{0}$ the central wave vector, and $\omega_{0}$ the central angular frequency. $T=$ $1+\left(i / \omega_{0}\right)(\partial / \partial t)$ is a differential time operator for spatiotemporal coupling [125]. $D$ is the dispersion coefficients at the center frequency which includes the effect of group velocity dispersion (GVD) and third-order dispersion (TOD), $c$ the speed of light and $n_{2}$ the nonlinear refractive index coefficient of the medium. The last three terms all belong to plasma generation process, where we apply Keldysh's multiphoton model for free electron density calculation, $\rho_{c}$ is the critical plasma density, $\beta^{(\kappa)}$ is the coefficient for multiphoton absorption, $\sigma$ the cross-section of collisional ionization, and the multiphoton ionization factor $\kappa$ is equal to 7 [99] under our conditions, including that the center wavelength is $800 \mathrm{~nm}$ and the medium is sapphire. If the laser intensity reaches $10^{13} \mathrm{~W} / \mathrm{cm}^{2}$, the photoionization rate must include contribution of tunneling ionization [117]. The plasma density $\rho$ in Eq. (1) satisfies the equation below which is widely applied in photon-ionization investigations:

$$
\frac{\partial \rho}{\partial t}=W_{I}\left(|U|^{2}\right)+\frac{\sigma \rho|U|^{2}}{U_{i}}-\frac{\rho}{\tau_{\text {rec }}},
$$

where $W_{I}$ is the photo-ionization rate, $U_{i}$ the ionization potential, and $\tau_{\text {rec }}$ the average time of electron recombination or attachment with neighboring ions. It is worth mentioning that $\tau_{\text {rec }}$ is considered because it has the same order of magnitude as the input pulse duration. To solve the NEE, we use a standard split-step Fourier algorithm treating dispersion in the frequency domain and the nonlinearity in the time domain. For the initial parameter setting, our general idea is to approximate experimental parameters as close as possible, which is also convenient for the subsequent training of the MLP neural network with combined experiment and simulation data, guaranteeing the prediction accuracy as well. We fix a 2-mm-thick sapphire as the nonlinear material in this paper. Although the laser-induced nonlinearities of different materials are of great interest as well, we decided to save this topic for a future discussion. Here, we set the initial pulse energy ranging from 10 to $30 \mu \mathrm{J}$, pulse spot radius from 100 to $200 \mu \mathrm{m}$ (on sapphire), and pulse duration from 30 to $100 \mathrm{fs}$, while they are close to the Fourier transform limit to eliminate the influence of pulse chirp. The spatiotemporal form of the input pulse is approximately a Gaussian shape; then the output spectrum is acquired numerically by the NEE simulation.

\section{QUANTIFICATION OF NONLINEAR EFFECTS}

In supercontinuum generation, the Kerr effect and ionization (plasma effect) are the two key nonlinear processes responsible for spectral broadening. In general, a broad output spectrum, especially symmetrically broadened, indicates a strong Kerr effect, while an asymmetric and blueshifted output spectrum implies a significant plasma effect and selfsteepening, as observed frequently in spectral broadening experiments. However, these judgments are imprecise and need to be improved. Unfortunately, the Kerr effect and ionization, as discussed earlier, are entangled with each other and cannot be measured or characterized separately in experiments. To gain profound insight into these two effects, we must rely on numerical simulations of the laser propagation with the NEE. Traditionally, a B integral [121] has been 

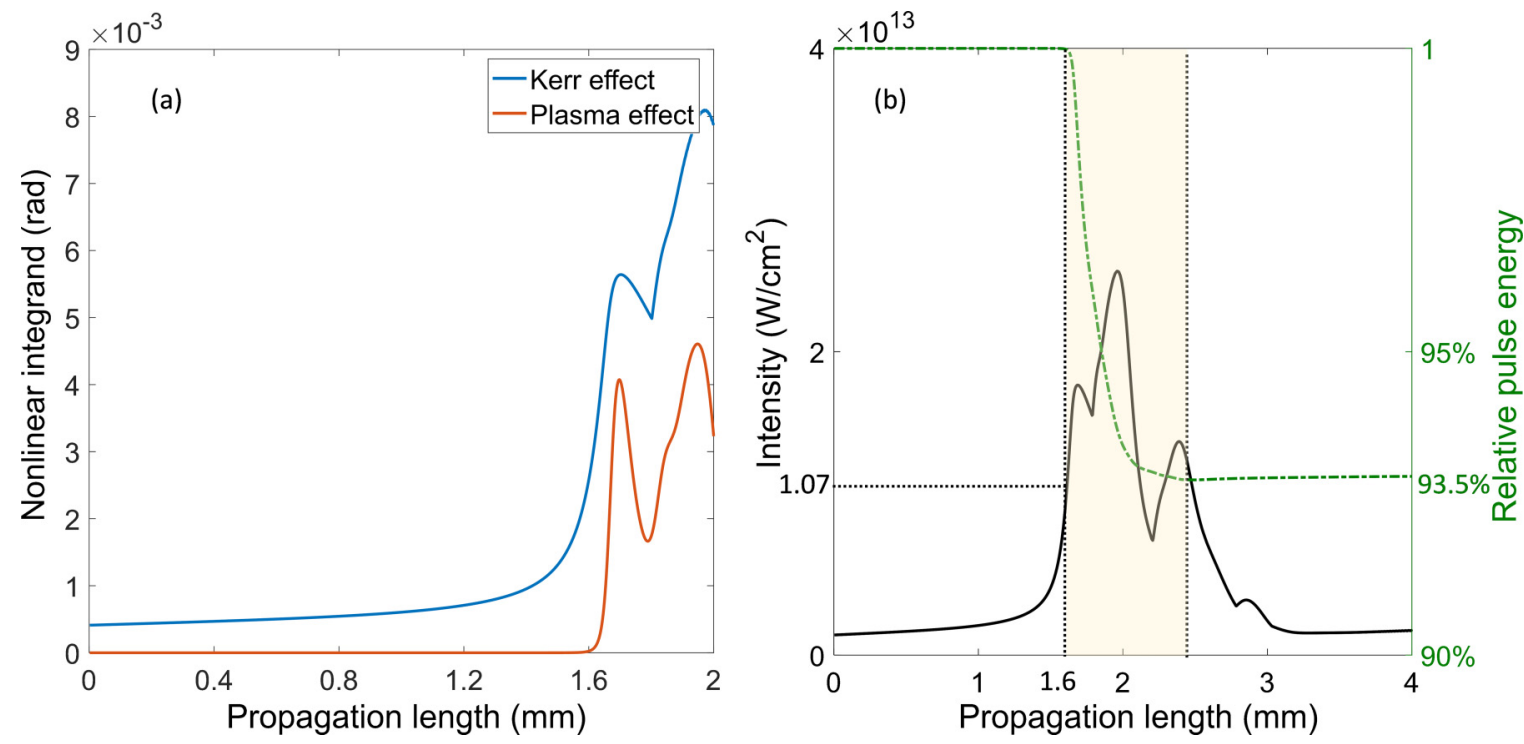

FIG. 1. (a) The numerically simulated integrands of $\mathrm{B}$ and $\mathrm{P}$ integrals ( $\Delta n_{\text {Kerr }}$ and $\Delta n_{\text {plasma }}$ ) as functions of pulse propagation distance. The input parameters are the pulse duration $\tau=40 \mathrm{fs}$, beam radius $r=0.1 \mathrm{~mm}$, pulse energy $E=0.02 \mathrm{~mJ}$, and focus length $f=150 \mathrm{~mm}$, and the $2 \mathrm{~mm}$ sapphire plate is set $5 \mathrm{~mm}$ before the focal point. Before the formation of the filament, plasma almost has no effect on pulse evolution, while the Kerr effect is not strong either. When optical clamping occurs $\sim 1.6 \mathrm{~mm}$, the strengths of both nonlinear effects increase sharply. (b) The variation of pulse intensity and energy with propagation distance. During the propagation, low and high ionization stages are experienced, and the high ionization stage is covered by the yellow shadow. The plasma density generated by the peak intensity is $\sim 1.63 \times 10^{15} \mathrm{~mm}^{-3}$.

defined to assess the nonlinear phase accumulation in laser amplifiers, which is an indicator of the strength or efficiency of the Kerr effect. In supercontinuum generation, the B integral also implies how wide the spectrum is broadened. The total change of the refractive index caused by the laser field has contributions from both the Kerr effect and ionization (plasma formation). To describe the overall nonlinear effect, a quantity to express the strength of ionization is necessary. Therefore, we define a $\mathrm{P}$ integral, like the $\mathrm{B}$ integral, to represent the phase change induced by plasma. They also provide a convenient and efficient method to bridge experimental data and numerical results, although not directly, and act as guidance for the control and optimization of these two nonlinear effects.

In general, the nonlinear effect is described in terms of its contribution to the refractive index $n=n_{0}+\Delta n_{\text {Kerr }}+$ $\Delta n_{\text {plasma }}=n_{0}+n_{2} I+\Delta n_{\text {plasma }}$, so the accumulated phase shift generated by Kerr effects along the laser beam axis during propagation is determined by the $\mathrm{B}$ integral:

$$
B_{\text {Kerr }}=\frac{2 \pi}{\lambda} \int_{0}^{z} \max \left(\Delta n_{\text {Kerr }}\right) d z=\frac{2 \pi n_{2}}{\lambda} \int_{0}^{z} I_{\max } d z .
$$

Similarly, the plasma induced phase change is expressed by a P-integral

$$
P_{\text {ion }}=\frac{2 \pi}{\lambda} \int_{0}^{z} \max \left(\Delta n_{\text {plasma }}\right) d z=-\frac{\pi e^{2}}{m c \omega_{0}} \int_{0}^{z} \rho_{\max } d z,
$$

and the variation of refractive index is deduced from the frequency shift caused by the ionized free electrons $\Delta n_{\text {plasma }}=$ $-\omega_{\rho}^{2} / 2 \omega_{0}^{2}=-2 \pi e^{2} \rho / m \omega_{0}^{2}$, where $e$ is the electron charge (positive value), $m$ the electron mass, and $\omega_{p}=\sqrt{4 \pi e^{2} \rho / m}$ the characteristic plasma angular frequency. Meanwhile, $I_{\max }$ and $\rho_{\max }$ are the peak intensity and peak plasma density, respectively, in the spatial-temporal space $(r, t)$ at each $z$ position. These two integrals are calculated as the NEE is numerically solved, and they are sufficient to distinguish and characterize Kerr and plasma effects.

Figure 1 shows the simulated integrands of $\mathrm{B}$ and $\mathrm{P}$ integrals, $\Delta n_{\text {Kerr }}$ and $\Delta n_{\text {plasma }}$, change as the pulse propagates in a sapphire plate. The input pulse parameters in the simulations are based on typical experimental conditions when a supercontinuum is acquired in a single filament formed in the solid medium. Firstly, Fig. 1(a) shows that, before filament occurs, the Kerr effect $\Delta n_{\text {Kerr }}$ plays the major role in the contribution to the nonlinear phase, and the plasma effect $\Delta n_{\text {plasma }}$ equals nearly zero because the pulse intensity is too weak to generate sufficient free electrons. Once filamentation is formed, plasma defocusing combined with diffraction balances self-focusing of the Kerr effect, and $\Delta n_{\text {plasma }}$ starts to rise quickly. Comparing the two peaks in Fig. 1(a), the vertical gap between the $\mathrm{B}$ and $\mathrm{P}$ integrands at the second peak is significantly wider than at the first one, which means the beam spot is smaller, and the magnitude of the diffraction effect is higher at the second peak. While Fig. 1(b) demonstrates the simulated pulse intensity variation and pulse energy attenuation as functions of pulse propagation length, we find that energy loss mainly occurs in the region where the $\mathrm{P}$ integrand rises up to the same order of magnitude as the Kerr effect (B integrand), which is also consistent with the energy loss in the NEE [the last two terms in Eq. (1)] being introduced by the plasma density. Meanwhile, in the shadow area in Fig. 1(b), where the plasma density is high, the pulse intensity drops quickly, and the energy loss stops at the end, implying where the filament takes place. Overall, the ionization takes a minor effect until the pulse propagates to $1.6 \mathrm{~mm}$, and 

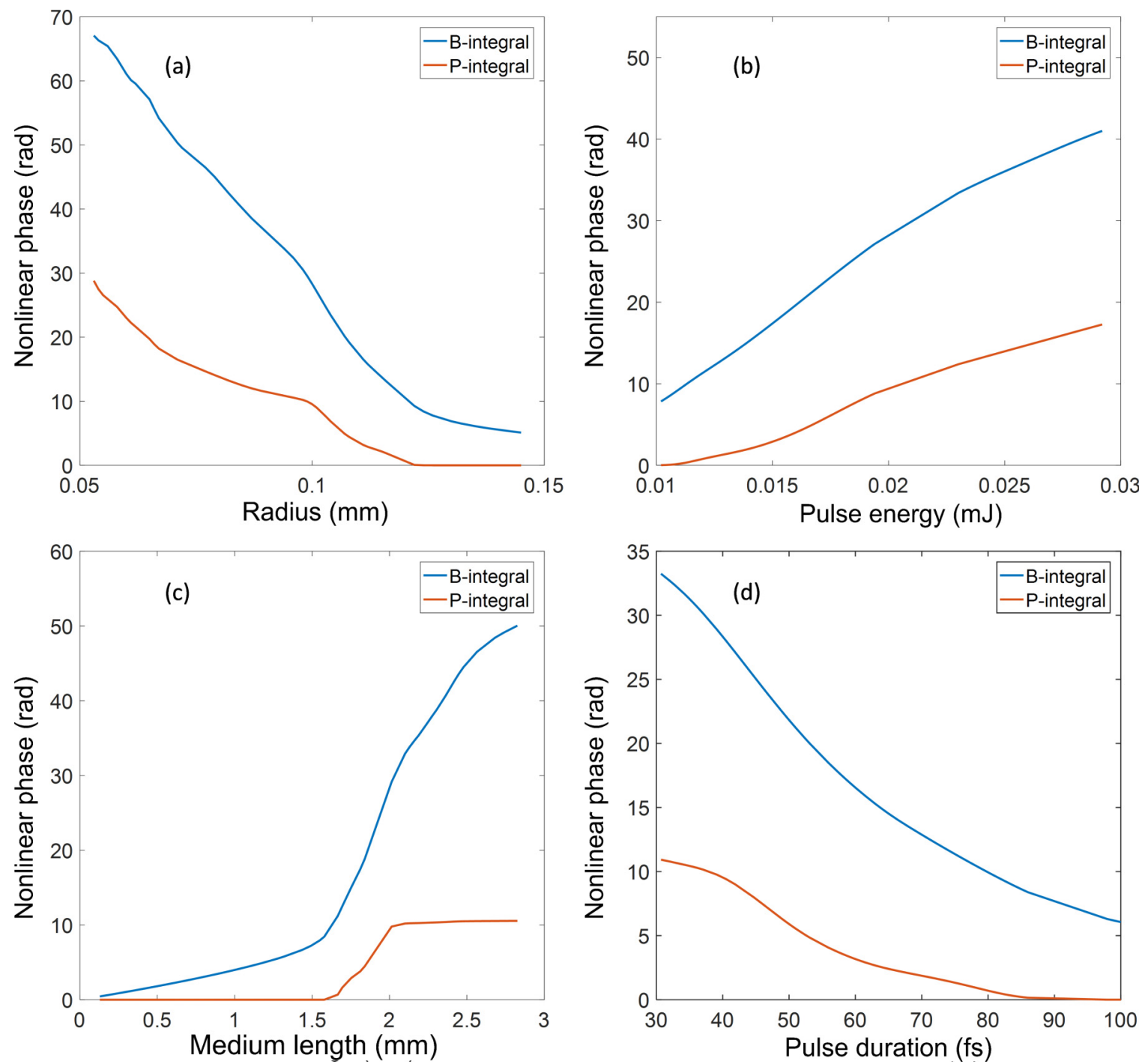

FIG. 2. Evolutions of B and P integrals as functions of (a) beam radius, (b) pulse energy, (c) medium length, and (d) pulse duration. When each single parameter changes in the simulation, other ones remain constant and the same as in Fig. 1(a).

the corresponding intensity is $1.07 \times 10^{13} \mathrm{~W} / \mathrm{cm}^{2}$, which is the threshold when the ionization starts to play a major role. Figure 1(a) shows similarly that the ionization rises sharply at $1.6 \mathrm{~mm}$.

Figure 2 shows evolutions of $\mathrm{B}$ and $\mathrm{P}$ integrals as different input parameters of the laser pulse change individually. When the input beam radius increases, both $\mathrm{B}$ and $\mathrm{P}$ integrals decrease simultaneously, as shown in Fig. 2(a). Once the radius becomes larger than a certain value $(\sim 0.12 \mathrm{~mm})$, the pulse intensity $\left(1.05 \times 10^{13} \mathrm{~W} / \mathrm{cm}^{2}\right)$ is not enough to generate plasma in the medium, and the Kerr effect becomes dominant. At this point, the low B-integral value also indicates weak spectral broadening. Meanwhile, as we discussed in the last paragraph about the vertical gap of $\mathrm{B}$ and $\mathrm{P}$ integrands, it is clear that the diffraction over the entire propagation becomes stronger with the increment of pulse energy in Fig. 2(b), foreboding a longer filamentation channel at higher pulse energy if filament occurs, while Fig. 2(d) reflects a similar physical mechanism with the change of input pulse width. Then the integrals as functions of the medium length reflect where the filament locates, as shown in Fig. 2(c). The $\mathrm{P}$ integral stays almost constant when the medium length increases beyond $2 \mathrm{~mm}$, which means optical clamping disappears at $2 \mathrm{~mm}$, where the pulse intensity is $9.7 \times 10^{12} \mathrm{~W} / \mathrm{cm}^{2}$. In general, this intensity ( $\sim 1 \times 10^{13} \mathrm{~W} / \mathrm{cm}^{2}$ under the condition in our simulation) is such a threshold that, when the pulse intensity is lower than this value, the Kerr effect is dominant, and the plasma density is not enough to have a significant effect on the broadening process ( $\mathrm{P}$ integral is almost zero). When the intensity is greater than this threshold, the $\mathrm{P}$ integral begins to accumulate significantly. The $\mathrm{P}$ integral provides a quantitative and distinct description of the plasma-induced effect, better than the plasma density itself, which is nonzero $\left(\sim 1.1 \times 10^{14} \mathrm{~mm}^{-3}\right)$ at this intensity.

Photon ionization in the spectral broadening process takes three stages with the growth of the laser pulse energy. At the first low ionization stage, the Kerr effect is dominant, and ionization is negligible. In the second high ionization stage, as the pulse intensity goes over $1 \times 10^{13} \mathrm{~W} / \mathrm{cm}^{2}$, the plasma effect increases rapidly and works in conjunction with the Kerr 

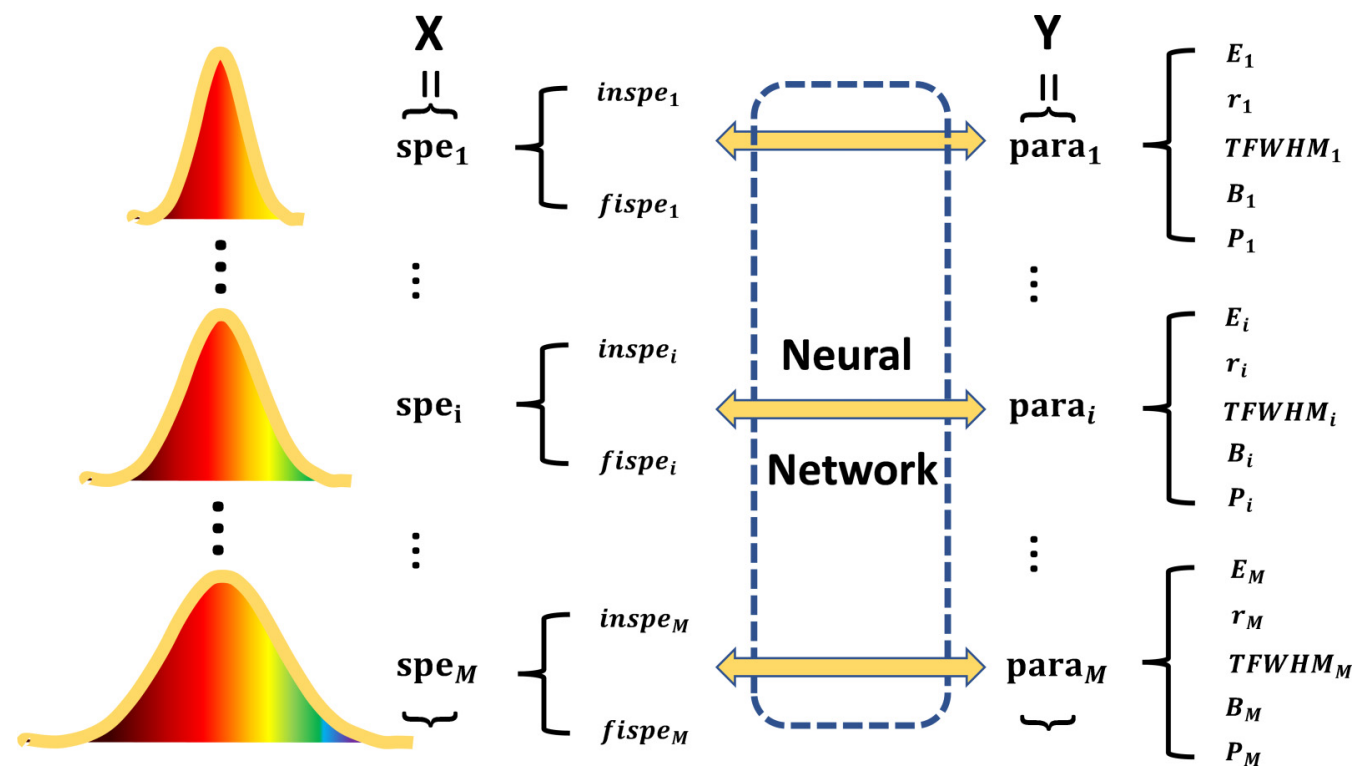

FIG. 3. The mapping relationship between the initial and final spectra and the laser propagation parameters.

effect to produce a series of nonlinear phenomena including filamentation. Finally, the avalanche ionization threshold is reached if the pulse intensity keeps growing, and the medium suffers irreversible ionization damage, which is the overionization stage.

The evolution of the two integrals as pulse duration varies shows that shorter pulses induce a stronger Kerr effect than longer ones under the same pulse intensity due to the steeper edges of the pulse envelop in time domain, as shown in Fig. 2(d). When a short pulse enters the medium, the magnitude of SPM is higher than a long pulse. Considering that the plasma effect sets in much later than the Kerr effect, as shown in Fig. 1(a), it appears that, before a short pulse experiences the plasma effect, SPM has already provided a substantial amount of spectral broadening. However, a long pulse has to go through a lengthy and weak SPM process until filament is acquired; thus, to get the same degree of spectral extension, a longer pulse suffers more of the plasma effect, which has already been proved to cause more phase uncertainty [126]. Therefore, short pulses cause less coherent loss in nonlinear propagation than long ones [127].

\section{MACHINE LEARNING AND NEURAL NETWORK}

In the field of machine learning, the MLP has been proved to be capable of approximating continuous functions [124]. Therefore, we employ a fully connected MLP to fit the procedure of solving the NEE numerically to simulate the spectral broadening process. In principle, the MLP network is capable of computing propagation parameters, only needing the initial and final spectra for the laser propagation through a piece of solid medium. To map the relationship between the input and output spectra and initial laser parameters as well as B and $\mathrm{P}$ integrals, the physical problem of pulse propagation needs to be adapted to match the framework of the MLP. We define $X=\left\{s p e_{1}, s p e_{2}, \ldots, s p e_{i}, \ldots, s p e_{M}\right\}$ as the input data in the training database for the MLP, where $M$ is the size of the database, $i$ is the index of the dataset in the database which goes from 1 to $M$, and $s p e_{i}$ including inspe and $f i_{s p e_{i}}$ represent the $i$ th set of pulse spectra before and after spectral broadening process and are one-dimensional vectors that contain 3000 elements each. The output data is defined as $Y=\left\{\operatorname{para}_{1}, \operatorname{para}_{2}, \ldots, \operatorname{para}_{i}, \ldots, \operatorname{para}_{M}\right\}$, where $\operatorname{para}_{i}=\left\{E_{i}, r_{i}\right.$, TFWHM$\left.M_{i}, B_{i}, P_{i}\right\}$. The items in $\operatorname{para}_{i}$ are, respectively, the initial pulse energy, beam spot radius, pulse duration, and the values of $\mathrm{B}$ and $\mathrm{P}$ integrals. All these items are scalar values indicating laser propagation characteristics, and the relationship between $X$ and $Y$ is shown in Fig. 3 . Machine learning is a data-driven process and requires a large training database to ensure the accuracy of computation. Here, we implement a two-stage method to build a mapping relationship between $X$ and $Y$.

At the first stage, we apply an autoencoder to reduce the size of the spectral data array to prevent overfitting and improve the speed of convergence in the following training process. The spectral data (arrays of 3000 elements) are encoded into feature vector arrays of five elements by an autoencoder network of eight encoder layers, as shown in Fig. 4. Then the encoded spectral data are introduced into the MLP network. After the training of the MLP, the encoded spectral data arrays $(1 \times 5)$ are decoded with a decoder network to restore the original data structure $(1 \times 3000)$. The network of the encoder and decoder is trained with 3000 spectra by unsupervised training separated from the training of the MLP network.

In the second stage, the encoded spectral data are introduced into the MLP. The MLP has three parts. The first is the input layer, which is mainly used to import and collate the encoded spectral data. Then the data are handed over to the hidden layers (256 nodes in each layer), and a series of fully connected operations are executed between every two layers to ensure the high accuracy of the network. Finally, the output layer composed of five nodes is used to calculate the $Y$ set as the output of the network. The structure of the two-stage neural network including the autoencoder and the MLP is shown in Fig. 5. 

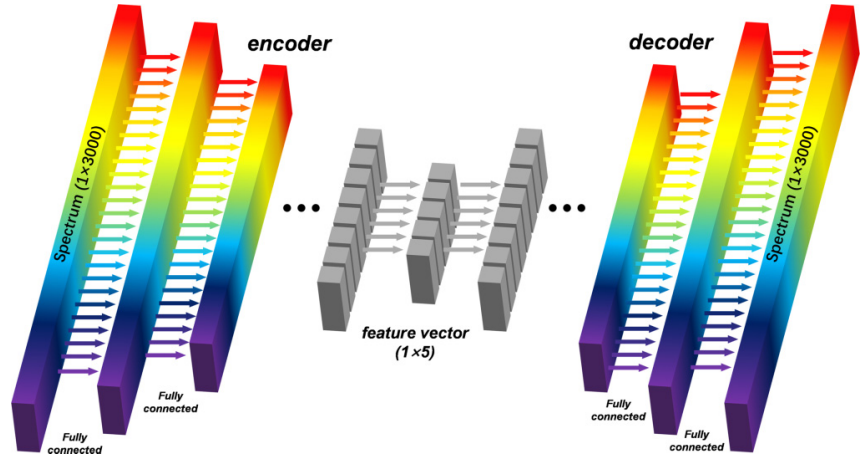

FIG. 4. Spectral data processing. The autoencoder is composed of an encoder and a decoder. The input of the encoder is a spectral data array. After passing through eight hidden layers, a feature vector with five elements is obtained. The input data of the decoder are the feature vector, and the spectral data array are restored after eight hidden layers.

For the activation function selection of the two-stage neural network, we abandon the traditional ones such as Sigmod, tanh, or ReLU but apply the sine function [128]. The main reason is those traditional functions are ineffective in simulating fine details and spatiotemporal coupling problems, while the sine function is found to be the most suitable in solving partial differential equations such as the NEE.

When training the network, 1800 sets of data calculated from the NEE are implemented, which are divided into training and testing subsets by a ratio of 95 to $5 \%$ randomly. In general, the ratio between training and testing subsets does not contribute to the final prediction results, but we find our prediction accuracy becomes worse when we raise the weight of the testing subsets, and this situation may be alleviated if we increase the total number of the datasets. In the training process, the division of all the data into training and testing subsets is executed once unless a new training program starts. However, the data in training subsets may be injected into the

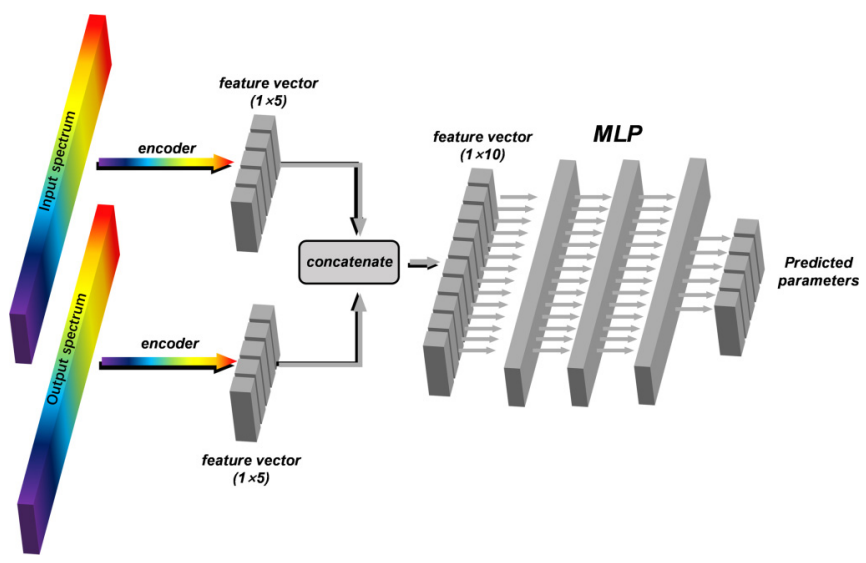

FIG. 5. Structure of the neural network. The original and broadened spectra pass through the same encoder and serve as the multilayer perceptron (MLP) input data. After three hidden layers, the predicted parameters as the output of MLP are the initial laser parameters (pulse width, beam size, and pulse energy) and the nonlinear $\mathrm{B}$ and $\mathrm{P}$ integrals. network several times, until the loss value calculated with the presorted testing subset does not decrease further. It is worth noting that the cost functions, which are the average of the loss functions of all the training data, of the abovementioned two stages are different because the training of the autoencoder is unsupervised while the MLP network training is supervised. For the first stage, the cost function is

$$
\operatorname{cost}_{a e}\left(\theta_{d}, \theta_{e}\right)=\sum_{0}^{M}\left|x^{(i)}-D_{\theta_{d}}\left\{E_{\theta_{e}}\left[x^{(i)}\right]\right\}\right|,
$$

where $\theta_{d}$ and $\theta_{e}$ are the tunable hyperparameters of the decoder and encoder, respectively, $D_{\theta_{d}}$ and $E_{\theta_{d}}$ are the output data calculated by the decoder and encoder, and $x^{(i)}$ is the $i$ th set of spectral data including the input and broadened spectra. After the network in the first stage is well trained, the cost function of the second stage is defined as

$$
\operatorname{cost}_{\mathrm{mlp}}(\theta)=\sum_{0}^{M}\left|y^{(i)}-\operatorname{MLP}_{\theta}\left\{E_{\theta_{e}}\left[x^{(i)}\right]\right\}\right| .
$$

The difference between these two cost functions is the reference parameter. For the autoencoder, the reference is the spectrum itself, while for the MLP, the target parameters $y^{(i)}$ in the mapping set $Y$ are applied as the reference. Finally, an Adam optimizer is used in the training, as it is one of the most widely used optimizers [129]. When we apply the numerical simulation results in the training of the neural network, the predictions are excellent, as shown in Fig. 6. The plots in Fig. 6 show the accuracy of prediction of each parameter computed by the network. The predicted values (orange circles) in all five plots fit well with the actual values (blue line) calculated in simulation, which means the predicting accuracy of a well-trained MLP is comparable with the numerical simulation of the NEE.

At the same time, we also investigate the ability of the trained network in out-of-range computation. In Fig. 7, the black dashed line is the value of initial pulse energy in the NEE simulation that we want the network to compute, while the orange filled area is the distribution of several values computed by the network trained with a database covering initial pulse energy from 0.01 to $0.03 \mathrm{~mJ}$. The results in Fig. 7 show that the computation of the network matches the target values in the simulation very closely in the training range, diverges from the target values, and exhibits an uncertainty up to $\sim 50 \%$ once the initial pulse energy goes beyond the training range. The uncertainty of the computation (the width of the orange band in Fig. 7) shows two features. First, the computations always follow the target energy values inside and outside the training data range. Second, the uncertainty decreases as the energy approaches the training range from the outside, and at 0.005 and $0.035 \mathrm{~mJ}(0.005 \mathrm{~mJ}$ away from the borders of the training range), the uncertainty drops to $\sim 25 \%$. These features indicate that, although the ability of a fully connected neural network to make computations outside its training range is limited, the computation results outside are still quite reasonable, and the closer to the training range, the better the result. 


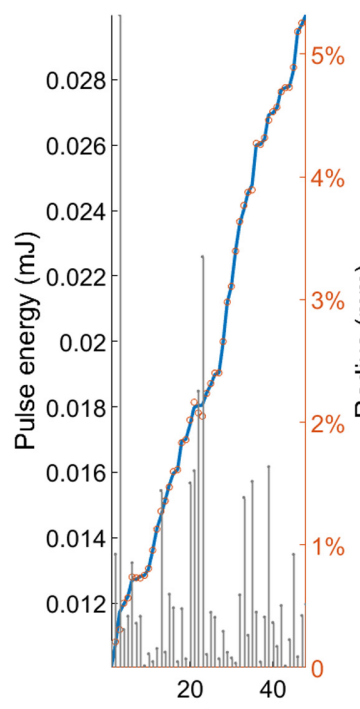

Index of dataset
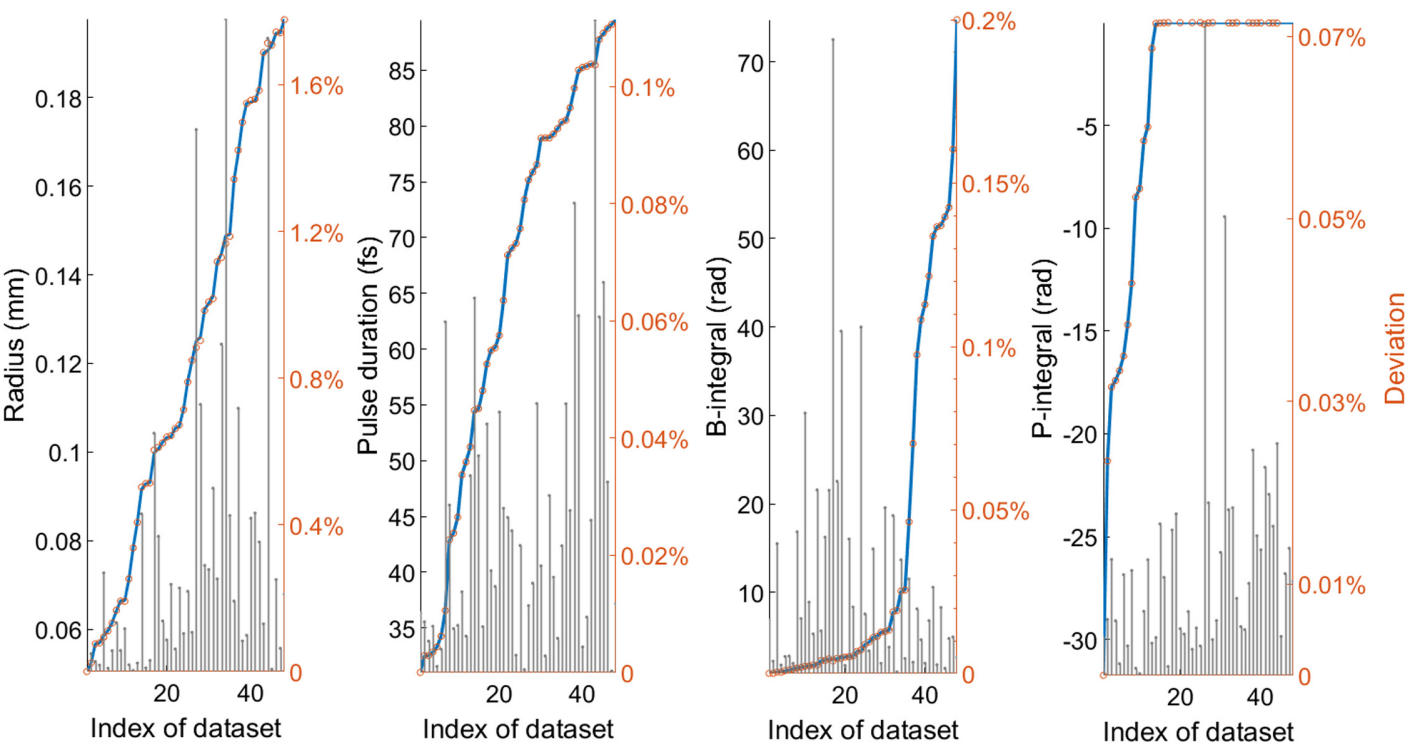

FIG. 6. Performance of the trained neural network in computing pulse energy, beam radius, pulse duration, and B and P integrals based on simulation dataset. The deviation between predicted value (orange circles) and simulated value (blue lines) is labeled on the right side of each plot, which reveals a high computation accuracy of all five parameters. The index of the dataset along the $x$ axis corresponds to the testing dataset chosen from the $X-Y$ database. Here, 50 datasets are selected as the testing set from the whole database randomly.

\section{EXPRIMENTAL MEASUREMENT OF LASER PARAMETERS AND SPECTRA}

To bring the MLP predictions closer to reality and obtain the unmeasurable $\mathrm{B}$ and $\mathrm{P}$ integrals from experiments for realworld observation of nonlinear propagation properties as well as obtain the initial pulse parameters for spectral broadening optimization in real time, we need to combine simulation and experiment data together to train the network and compute laser parameters from experimental data. Through this operation, the propagation process can be traced back by the neural network, and the information of the initial pulses and their experienced nonlinearity can be extracted from the experimental spectra, which is impossible in a standard propagation equation solution and is particularly suited in spectroscopic studies of laser-matter interaction. The training data acquired from experiments ensure the accuracy of subsequent experimental parameter prediction with the presence of experimental noise, which is crucial in neural network redress.

We obtain relevant pulse and spectral data in experiments under the same conditions as we use in the numerical simulations of the NEE. The experimental setup is shown in Fig. 8. The laser system is a homebuilt $\mathrm{Ti}$ : sapphire regenerative amplifier [130], and we use a polarization attenuator to control the pulse energy. A 2-mm-thick sapphire plate is used as the nonlinear medium, which is placed on a translation stage to adjust the beam spot size on it. We choose sapphire instead of fused silica because sapphire has a larger $n_{2}$ so that the nonlinear effect is stronger under the same laser condition. The sapphire plate is cut perpendicular to the crystal axis, and we keep the pulses propagating along the crystal axis to avoid birefringence. A pair of wedges are inserted before the sapphire plate for beam sampling, and the reflection of the first surface is sent to a charge-coupled device (CCD; DataRay) to measure the beam radius. Here, we mark the relative position of the sapphire plate to the focal point and put the CCD at the same distance to ensure the real beam radius on the plate is measured by the CCD. Meanwhile, we use a Dazzler (Fastlite) to accurately control the pulse duration and measure the pulse width (and temporal shape) by a Wizzler (Fastlite). The spectra are taken by a spectrometer (Ocean Optics). By measuring the input pulse energy, duration, beam radius on the sapphire plate, and spectra before and after the sapphire plate, we collect all the quantities necessary to compare with the numerical simulation and to establish experimental datasets for the training of the MLP network. The laser conditions are pulse energy 10 to $30 \mu \mathrm{J}$, pulse width 30 to $100 \mathrm{fs}$, and beam radius 100 to $200 \mu \mathrm{m}$ on the sapphire plate. In experiments, we find that the laser power instability is $\sim 1 \%$ measured by a power meter (Coherent) over $15 \mathrm{~min}$, the uncertainty of pulse width measurement is $\sim 3 \mathrm{fs}$, and for beam radius, the uncertainty is $\sim 20 \mu \mathrm{m}$. When the experimental data are

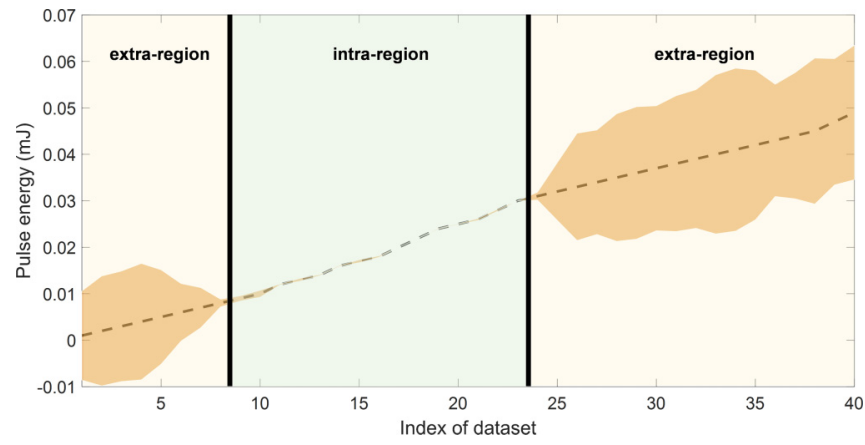

FIG. 7. Computation of the target parameters in an extended range. Here, we take pulse energy as an example. The orange filled area shows the computation uncertainty. The computed values match well with target values in the training data range $(0.01$ to $0.03 \mathrm{~mJ})$, and the computation uncertainty increases when the energy moves out of the training range. 


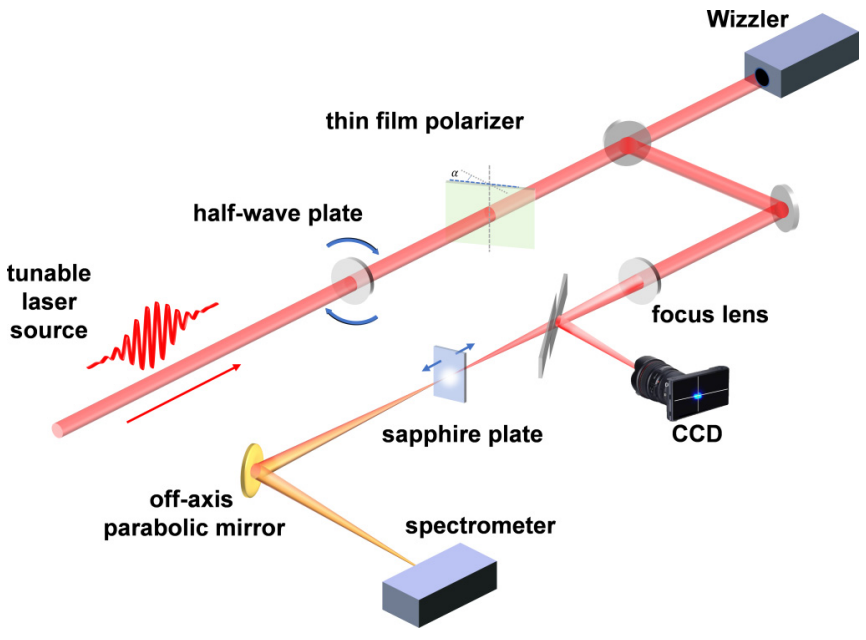

FIG. 8. The schematic diagram of the experimental setup to collect initial laser pulse parameters as well as spectra before and after the nonlinear medium. The optics to measure the spectrum before the nonlinear medium are not shown. HWP: half-wave plate, TFP: thin film polarizer, BS: beam splitter, PM: plane mirror, FL: $f=150 \mathrm{~mm}$ focus lens, WP: wedge pair, SP: sapphire plate, OAP: off-axis parabolic mirror, CCD: camera to measure beam size, Wizzler: device to measure pulse width. In principle, there is no special requirement to the input laser pulse in experiments if it generates spectral broadening in the nonlinear medium. However, considering the consistency of parameters with the numerical simulations and reasons discussed in the text, we set the input pulse to be close to the Fourier transform limit.

used in the training, the uncertainties will finally propagate into the uncertainty in the computations of the neural network. In the experiments, we totally obtain $\sim 350$ sets of data. Although it is hard to quantify how the measurement uncertainty affects the training, when training the network with experiment data only, the result is far from ideal. The main reasons are insufficient number of datasets and the noise in the measurement, resulting in misfitting of the network. Since there is no uncertainty in the simulation, training with both experimental and simulation data resolves this issue. At the same time, to avoid overfitting with the simulation data only and improve the accuracy of experimental prediction, including experimental data in the training database is necessary. Among all the parameters of femtosecond pulses, it is always difficult to measure the spectral phase in experiments. All the phase measurements are indirect, and numerical iterative algorithms are usually involved to retrieve the values of the phase, which makes the uncertainty of the phase measurement not only large but hard to be estimated as well. Therefore, we decided to use only the spectra before and after the nonlinear medium as the input parameters for the MLP network and abandon the spectral phase for the time being. It is generally understood and reported by previous researchers that, without the phase information, machine learning can still make successful predictions with spectral information only [131]. We also take transform-limited or close to transform-limited pulses as the input for both experimental and simulation data collections to further reduce the influences of the spectral phase in this paper. Meanwhile, it is crucial in the training

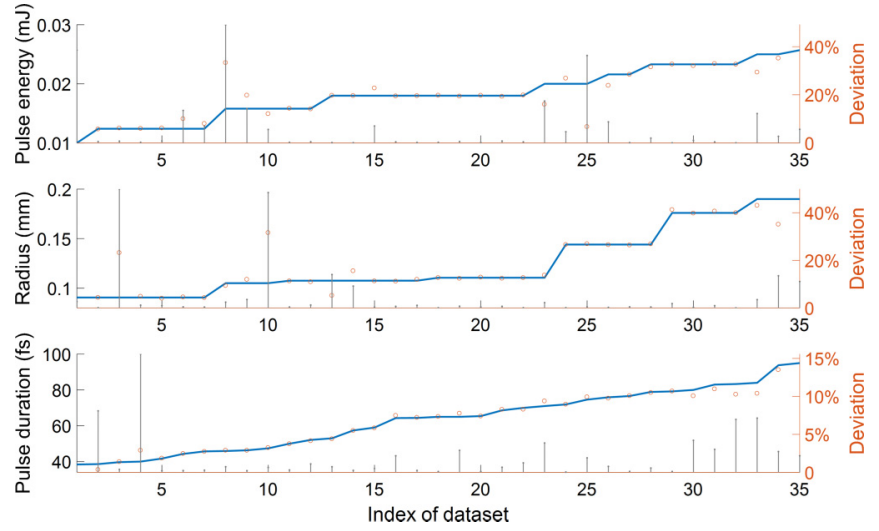

FIG. 9. Computing the initial pulse parameters from the experimentally measured spectra. The computed values (orange circles) fit well with reference experimental data (blue curves), especially the results of pulse duration. The reference data are extracted from the 350 sets of experimental data randomly and are not included in the training dataset for the network to ensure reliability.

process that the spectral width is given more weight than the exact spectral shape to make sure the fitting precision for both experiment and simulation data because details of the spectral shape are subject to the noise and uncertainty in the experiments and numerical calculations, causing overfitting in the training process. In summary, hybrid data are used in our training database to map the relationship between initial pulse properties and spectra before and after the solid medium, where the simulation part is for data-deficient recoupment and experimental part for network redress.

Finally, we combine simulation and experiment data together to train the network and compute laser parameters from the reference experimental datasets. The computed and measured values are shown together in Fig. 9, which shows clearly that our trained network has computed the values successfully. The computed pulse duration has the best performance among all the parameters, mainly because of its larger training database. In principle, if we could have collected enough data in experiments to train a more sophisticated network, we may be able to employ such a network in femtosecond pulse temporal measurement due to its accurate computation of the pulse width. Moreover, new parameters may be incorporated into the network and the training database so that the network may compute more characteristic values of the laser pulse and propagation. However, in this paper, the accuracy of the computation of experimental data is not as good as that for simulation data. There are two major issues. One is that the NEE does not consider higher order nonlinearities so that the numerical result does not simulate the actual experimental situation completely. The other is that the uncertainty and noise in experimental measurement are not considered in the MLP network. Solving these issues is the goal of our subsequent work.

Although it is impossible to establish a training database of nonlinear effects experimentally because $\mathrm{B}$ and $\mathrm{P}$ integrals cannot be directly measured, we have constructed the relations between spectra and two main spectral broadening processes with numerical simulations. Combining both simulation and 


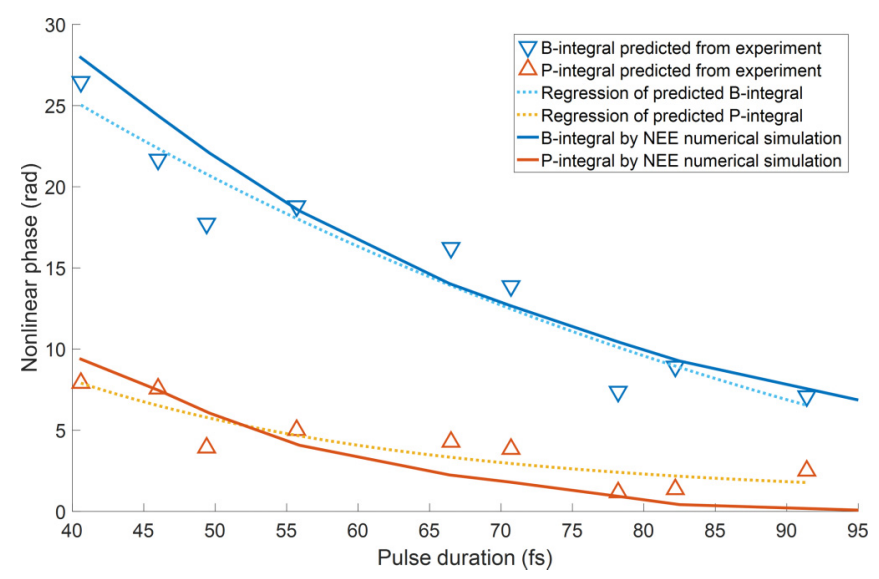

FIG. 10. Computing the nonlinear phases ( $\mathrm{B}$ and $\mathrm{P}$ integrals) from experimental spectra, which are collected under the laser conditions where the pulse energy is $21 \mu \mathrm{J}$, beam radius is $0.1109 \mathrm{~mm}$, and pulse width changes from 40 to $95 \mathrm{fs}$. B and $\mathrm{P}$ integrals simulated by the nonlinear envelope equation (NEE) are plotted in comparison. The computed and simulation results as functions of pulse width are consistent.

experiment data in the training database is successful for this preliminary verification, as we have shown here, and provides a solid foundation for future applications of the MLP network in experiments to achieve real-time computation of the nonlinear parameters. From the perspective of the neural network structure, the computation of initial pulse parameters and $\mathrm{B}$ and $\mathrm{P}$ integrals are intercoupled. It is easy to understand that all the parameters inside a fully connected neural network are linked with each other by the hidden layers, which means, when we apply experimentally measured data (pulse energy, beam radius, and pulse width) in a well-trained network whose original training data are from simulation, the network is modified, and the computation gets closer to reality. This procedure will not only affect the computation of the experimental parameters but also the $\mathrm{B}$ and $\mathrm{P}$ integrals. The computation accuracy of initial pulse parameters, as shown in Fig. 9, reflects the accuracy of the two nonlinear integrals indirectly, so that the computation of $\mathrm{B}$ and $\mathrm{P}$ integrals is also reliable.

Figure 10 shows the evolution of computed nonlinear phases ( $\mathrm{B}$ and $\mathrm{P}$ integrals) as the pulse width changes. These two integrals are in fact functions of laser intensity, which is measured indirectly via pulse energy, pulse width, and beam radius in the experiments. While keeping the pulse energy and beam radius unchanged, we plot the nonlinear integrals as functions of pulse width. Although the integrals cannot be measured experimentally, a well-trained MLP network can compute the integrals from measured spectra so that a comparison with numerical simulation results is made, as shown in Fig. 10. The regression curves of computed data points match well with the simulation curves, which means that we have successfully quantified Kerr and plasma effects as the major nonlinearities in supercontinuum generation by the $\mathrm{B}$ and $\mathrm{P}$ integrals and obtained their values via experimentally measured spectra by a trained MLP network for the first time. These nonlinear integrals are effective tools to guide the optimization of the spectral broadening process and to analyze the output spectral phase, so that a balancing point between the wider spectrum and better phase condition may be sought in experiments.

\section{SHAPE OF THE BROADENED SPECTRA}

In supercontinuum generation, as the laser intensity rises (by either raising the pulse energy or shortening the pulse width), the output spectrum changes from a Gaussian form to an irregular shape which is broadened on both the long-wavelength [or low frequency (LF)] side and the shortwavelength [or high frequency $(\mathrm{HF})]$ side. Here, we analyze a simulated spectrum integrated within a central zone of $<0.1 \mathrm{~mm}$ around the beam axis. The central integrated spectrum is used for the comparison with the experimental spectrum, which is acquired by taking a small part of the beam near the center into the spectrometer. How the broadening occurs depends on the nonlinear effects the pulse experiences as it propagates through the medium. In general, SPM causes symmetric spectral broadening, while self-steepening and the plasma effect cause asymmetric broadening to the HF side. As our MLP network is trained with simulated and experimental data, a relationship between the output spectrum and the nonlinear effects in the propagation is established and encoded in the network so that, as we showed earlier, the network can compute the nonlinear integrals from the spectrum. To quantify such a relationship, we evaluate the frequency widths of the spectrum and integrated spectral densities (ISDs), which are the total signal strengths on the LF or HF sides, as well as the nonlinear integrals as the input pulse energy varies, and plot the results in Fig. 11.

As the pulse energy increases, the output spectrum is broadened on both HF and LF sides. The HF (LF) frequency width is measured from the central frequency $(3.75 \times$ $10^{14} \mathrm{~Hz}, 800 \mathrm{~nm}$ ) to the point where the density of the spectrum drops to $10^{-3}$ of the peak value. Figure 11(a) shows that the LF width rises at almost a constant slope, while the growth of the HF width appears to have a knee point $<0.02 \mathrm{~mJ}$, which implies that the spectral broadening by ionization has reached a threshold. Beyond this threshold, ionization causes significant instability in the phase of broadened spectrum [132,133]. At the threshold, the P integral equals $9.66(\approx 3 \pi)$, as shown in Fig. 11(b). To avoid instability of the broadened spectrum, the $\mathrm{P}$ integral needs to be kept below this value in few-cycle pulse generation setups, and this P-integral threshold could be a guide in design and operation of a spectral broadening system like the required specific B-integral values in fiber amplifiers $[121,134,135]$ and spectral broadening by a multipass cell [136]. When the pulse energy goes $>0.02 \mathrm{~mJ}$, although the HF width increases more slowly, as shown in Fig. 11(a), the $\mathrm{P}$ integral still grows at almost the same speed, as shown in Fig. 11(b), indicating that the nonlinear phase shift from the ionization effect after this point does not contribute to spectral broadening as much as before.

To further investigate the spectral change at the abovethreshold stage, we calculate the ratio of ISDs of the HF and LF sides, shown as the dashed curve in Fig. 11(b). It is clear that, below the threshold, the ISDs of both sides are roughly the same, and this part is identified as the first stage, where the densities of the HF and LF sides grow with similar 

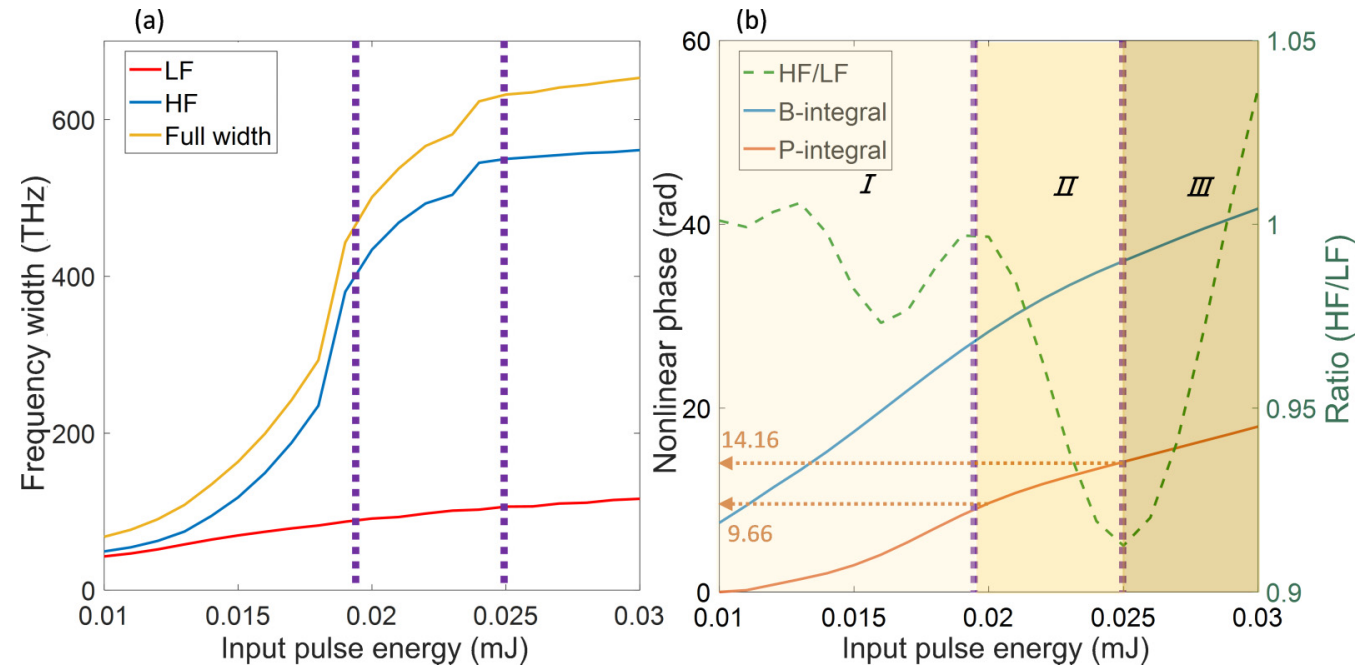

FIG. 11. Spectrum broadening as the pulse energy rises. (a) The frequency width variation of the final output spectrum. (b) The nonlinear integrals and the ratio of integrated spectral densities of the high frequency (HF) and low frequency (LF) sides as functions of the input pulse energy.

speeds and the LF density is slightly larger, while the HF width extends further, as shown in Fig. 11(a). In the second stage, the extension of HF is restrained, and the growing of LF dominates. Finally, as the input pulse energy goes $>0.025 \mathrm{~mJ}$ (and the P integral goes $>14.16$, which is $\approx 4.5 \pi$ ), the HF density increases sharply in the third stage, which is probably due to a large increase of ionization.

A careful comparison between Figs. 11(a) and 11(b) implies that the spectral shapes in the three stages are quite distinct. The spectrum is more or less symmetric in the first stage. In the second stage, the LF side extends more and gains more density as well so that the spectrum looks broader and heavier on the LF side. In the third stage, the LF side keeps extending, but the HF side gains more density so that the spectrum still looks broader on the LF side but heavier on the HF side. This evolution of the spectral shape is consistent with common observations in spectral broadening experiments when the pulse energy or intensity is increasing. However, these two nonlinear integrals, especially the $\mathrm{P}$ integral, provide more detailed information about how the spectral broadening takes place and is affected by ionization, and they are effective tools to analyze, quantify, and optimize the spectral broadening process.

\section{COMPARISON BETWEEN THE NEE AND THE MLP}

Solving the NEE with the split-step method is fundamental and the most reliable way to study pulse propagation numerically, and the MLP method is unable to replace the NEE completely because the mapping between the spectra and nonlinearities is constructed by the NEE, and most of the training data of the MLP is generated by the NEE. Meanwhile, the range of MLP prediction is also determined by the range of the training data produced by NEE simulations. Although the neural network will perform better when a more flexible model and a larger training database are employed, the large number of calculations required to construct such a training database may bring a great computational burden. However, the MLP method has several unique features that the NEE does not.

Firstly, the MLP network has high computing efficiency, and it takes only a few milliseconds for the MLP to compute a single propagation process on a regular office computer, much shorter than hours of solving the NEE. Even though it takes a large amount of time to collect training data and to train the network, the expense is restrained and controllable. Once the range of the laser parameters is defined, the collection of training data is straightforward with parallel computing (the calculation of 1800 groups of data on a super cloud computing platform lasts for $\sim 2$ days without human supervising), and the experimental data collection could be processed faster by programed electronic control equipment. Our well-trained MLP was built within 4 days; after that, the MLP may be employed in calculations of the spectral broadening process with a sapphire plate if the parameters are within the training range. Without the MLP network, a new numerical solution of the NEE is required every time the parameters are changed. Therefore, the construction of the neural network for propagation prediction is once for all. The high speed of the network prediction could support real-time feedback in experiments, which will contribute significantly in experimental research of ultrafast spectroscopy.

Secondly, it is difficult to introduce noise and higher order nonlinearities in a standard NEE, and approximations have been made in the deduction of the NEE, so that it is not reliable to match the NEE simulation results with experimental measurement. On the contrary, the neural network is data driven and can combine the simulation results from the NEE with experimental data, so the MLP may push the simulation results closer to reality. Under a certain pulse propagation condition, with experimental data in the training database, the MLP method is more realistic and accurate than the NEE.

Meanwhile, as discussed in Sec. V, the B and P integrals as well as pulse initial parameters are contained implicitly in the shape of the broadened spectrum. Only with a neural network may one extract such information while the traditional NEE 
is unable to do this kind of inverse calculation. Our result showing the prediction of the MLP is consistent with experimental data (Figs. 9 and 10), indicating that a well-trained MLP can help us investigate a certain laser-matter interaction experimental system with measured spectra only and quantify the implicitly involved nonlinearities. This is what the NEE cannot achieve, as it can only calculate the nonlinear integrals and the final spectrum from the initial pulse conditions.

\section{CONCLUSIONS}

In summary, we define a $\mathrm{P}$ integral, like the $\mathrm{B}$ integral commonly used in laser amplification and nonlinear optics, to illustrate the change of nonlinear refractive index and phase induced by plasma as well as the strength of ionization. The $\mathrm{P}$ and $\mathrm{B}$ integrals describe, characterize, and compare the two key processes, ionization and the Kerr effect, with each other in supercontinuum generation or as short laser pulses propagate through a medium in general. We perform numerical simulations to solve the $2 \mathrm{D}+1 \mathrm{NEE}$ to predict the final output spectrum as well as calculate the integrals for the propagation of femtosecond laser pulses through a solid medium. In our numerical simulations, by comparing these two integrals as the laser pulses propagate through the medium and by comparing the integrals as we vary the laser parameters, we find that the Kerr effect sets in to broaden the spectrum earlier in the propagation and under lower laser intensity; as the intensity goes over $\sim 1 \times 10^{13} \mathrm{~W} / \mathrm{cm}^{2}$, the plasma effect increases rapidly while causing significant energy loss. The numerical results also show that the Kerr effect provides a substantial amount of spectral broadening before the laser pulse starts to experience the plasma effect if the input pulse is short enough, indicating that a short pulse is advantageous in spectral broadening and to avoid the plasma effect comparing with a longer one. The $\mathrm{P}$ integral along with the traditional B integral provides a practical method to investigate, distinguish, and quantify the hidden and entangled nonlinear processes in supercontinuum generation with much better clarity.

Furthermore, we employ a fully connected neural network to fit the procedure of solving the NEE numerically to simulate the spectral broadening process to establish a mapping relationship between input and broadened spectra and initial laser parameters as well as B and P integrals as femtosecond pulses propagate through a piece of solid medium. We use datasets from both experiments and simulations for the training of the MLP network to avoid overfitting and improve the accuracy of real-time computations in experiments. A welltrained MLP network can find propagation parameters, only needing the spectra of the laser pulses, and the computation is almost instant. In experiments, this capability provides not only a scheme to characterize the input pulse in real time but a guide to optimize the supercontinuum generation process. Such a neural network can also compute parameters that cannot be measured experimentally. Through the network structure, the measurable parameters are connected with the unmeasurable ones, which provides a rigorous mechanism for us to estimate the unmeasurable parameters, such as the nonlinear integrals, of a physical process to gain deeper insights of the intermediate states not accessible before. Meanwhile, compared with the standard procedure of solving the NEE numerically, the neural network may push the simulation results closer to reality by including experimental results in the training database and predict the target parameters from input data regardless of the causal relationship between the input and output of the network. Additionally, the ultrafast computing speed and high accuracy ensure the reliability and uniqueness of MLP networks in experimental applications.

As an example, we investigate the spectral shape and the two nonlinear integrals with the MLP network as the input pulse energy increases. The results reproduce our common observation in spectral broadening experiments and show that the variation of the spectral shape is related to the increase of the integrals. These two nonlinear integrals, especially the $\mathrm{P}$ integral, provide detailed information about how the spectral broadening takes place and is affected by ionization. The MLP network is much faster in the calculation of the integrals than solving the NEE. Combined with the integrals, the network can provide effective ways to analyze, quantify, and optimize the spectral broadening process. The neural network introduced here may be considered as an extension and optimization of the NEE.

This approach, employing neural networks to fit the procedure of solving the NEE numerically to investigate spectral broadening or other nonlinear optical processes, provides a perspective in laser-matter interaction research and will have a significant impact on the research and applications of spectral broadening, supercontinuum and few-cycle pulse generation, as well as nonlinear and ultrafast optics in general.

\section{ACKNOWLEDGMENTS}

We thank Xi Long of Suzhou Intelligence Health InnoCentive, China, for helpful discussion and technical assistance in neural network theory and computer program coding and Dr. Tingting $\mathrm{Xi}$ of the University of Chinese Academy of Sciences for helpful discussion in solving the propagation equation. All numerical calculations in this paper were done at Beijing Super Cloud Computing. This paper was supported by the National Key Research and Development Program of China (Grant No. 2017YFB0405202) and the National Natural Science Foundation of China (Grants No. 61690221, No. 91850209, and No. 92150103).
[1] G. Mourou, S. Mironov, E. Khazanov, and A. Sergeev, Single cycle thin film compressor opening the door to zeptosecondexawatt physics, Eur. Phys. J. Spec. Top. 223, 1181 (2014).

[2] M. Chini, K. Zhao, and Z. H. Chang, The generation, characterization and applications of broadband isolated attosecond pulses, Nat. Photonics 8, 178 (2014).
[3] L. Y. Peng, W. C. Jiang, J. W. Geng, W. H. Xiong, and Q. H. Gong, Tracing and controlling electronic dynamics in atoms and molecules by attosecond pulses, Phys. Rep. 575, 1 (2015).

[4] T. Gaumnitz, A. Jain, Y. Pertot, M. Huppert, I. Jordan, A. L. Fernando, and H. J. Worner, Streaking of 43-attosecond 
soft-x-ray pulses generated by a passively CEP-stable midinfrared driver, Opt. Express 25, 27506 (2017).

[5] J. Li, X. M. Ren, Y. C. Yin, K. Zhao, A. Chew, Y. Cheng, E. Cunningham, Y. Wang, S. Y. Hu, Y. Wu et al., 53-Attosecond X-ray pulses reach the carbon $K$-edge, Nat. Commun. 8, 186 (2017).

[6] H. Mashiko, S. Gilbertson, C. Li, E. Moon, and Z. H. Chang, Optimizing the photon flux of double optical gated high-order harmonic spectra, Phys. Rev. A 77, 063423 (2008).

[7] F. Ferrari, F. Calegari, M. Lucchini, C. Vozzi, S. Stagira, G. Sansone, and M. Nisoli, High-energy isolated attosecond pulses generated by above-saturation few-cycle fields, Nat. Photonics 4, 875 (2010).

[8] M. Chini, X. Wang, Y. Cheng, Y. Wu, D. Zhao, D. A. Telnov, S. I. Chu, and Z. H. Chang, Sub-cycle oscillations in virtual states brought to light, Sci. Rep. 3, 1105 (2013).

[9] G. V. Marr and J. B. West, Absolute photoionization crosssection tables for helium, neon, argon, and krypton in the VUV spectral regions, Atom. Data Nucl. Data Tables 18, 497 (1976).

[10] J. Samson and W. C. Stolte, Precision measurements of the total photoionization cross-sections of $\mathrm{He}, \mathrm{Ne}, \mathrm{Ar}, \mathrm{Kr}$, and $\mathrm{Xe}$, J. Electron Spectrosc. Relat. Phenom. 123, 265 (2002).

[11] T. Sato, A. Iwasaki, K. Ishibashi, T. Okino, K. Yamanouchi, J. Adachi, A. Yagishita, H. Yazawa, F. Kannari, and M. Aoyma, Determination of the absolute two-photon ionization cross section of He by an XUV free electron laser, J. Phys. B 44, 161001 (2011).

[12] I. J. Kim, C. M. Kim, H. T. Kim, G. H. Lee, Y. S. Lee, J. Y. Park, D. J. Cho, and C. H. Nam, Highly Efficient HighHarmonic Generation in an Orthogonally Polarized Two-Color Laser Field, Phys. Rev. Lett. 94, 243901 (2005).

[13] P. Corkum, N. Burnett, and M. Ivanov, Subfemtosecond pulses, Opt. Lett. 19, 1870 (1994).

[14] G. Yang and Y. R. Shen, Spectral broadening of ultrashort pulses in a nonlinear medium, Opt. Lett. 9, 510 (1984).

[15] R. R. Alfano, The Supercontinuum Laser Source, 3rd ed. (Springer-Verlag, New York, 2016).

[16] F. Shimizu, Frequency Broadening in Liquids by a Short Light Pulse, Phys. Rev. Lett. 19, 1097 (1967).

[17] R. R. Alfano and S. L. Shapiro, Observation of SelfPhase Modulation and Small-Scale Filaments in Crystals and Glasses, Phys. Rev. Lett. 24, 592 (1970).

[18] P. B. Corkum, P. P. Ho, R. R. Alfano, and J. T. Manassah, Generation of infrared supercontinuum covering 3-14 $\mu \mathrm{m}$ in dielectrics and semiconductors, Opt. Lett. 10, 624 (1985).

[19] T. Brabec and F. Krausz, Intense few-cycle laser fields: frontiers of nonlinear optics, Rev. Mod. Phys. 72, 545 (2000).

[20] T. Nagy, P. Simon, and L. Veisz, High-energy few-cycle pulses: post-compression techniques, Adv. Phys. X 6, 1845795 (2021).

[21] D. W. McCamant, P. Kukura, S. Yoon, and R. A. Mathies, Femtosecond broadband stimulated Raman spectroscopy, Rev. Sci. Instrum. 75, 4971 (2004).

[22] M. Bradler and E. Riedle, Temporal and spectral correlations in bulk continua and improved use in transient spectroscopy, J. Opt. Soc. Am. B 31, 1465 (2014).

[23] D. J. Jones, S. A. Diddams, J. K. Ranka, A. Stentz, R. S. Windeler, J. L. Hall, and S. T. Cundiff, Carrier-envelope phase control of femtosecond mode-locked lasers and direct optical frequency synthesis, Science 288, 635 (2000).
[24] G. Humbert, W. J. Wadsworth, S. G. Leon-Saval, J. C. Knight, and D. Stifter, Supercontinuum generation system for optical coherence tomography based on tapered photonic crystal fibre, Opt. Express 14, 1596 (2006).

[25] H. D. Huang, C. Y. Hu, H. J. He, H. Teng, Z. Y. Li, K. Zhao, and Z. Y. Wei, Broadband mid-infrared pulse via intra-pulse difference frequency generation based on supercontinuum from multiple thin plates, Chin. Phys. B 28, 114203 (2019).

[26] J. Zhang, K. Fritsch, Q. Wang, F. Krausz, K. F. Mak, and O. Pronin, Intra-pulse difference-grequency generation of midinfrared (2.7-20 $\mu \mathrm{m})$ by random quasi-phase-matching, Opt. Lett. 44, 2986 (2019).

[27] Q. Wang, L. Qi, R. Y. Wang, and Y. Li, Research progress of mid infrared laser via intra-pulse difference frequency generation of femtosecond laser [in Chinese], Laser Optoelectron. Prog. 58, 1700001 (2021).

[28] M. K. Reed, M. K. Steiner-Shepard, M. S. Armas, and D. K. Negus, Microjoule-energy ultrafast optical parametric amplifiers, J. Opt. Soc. Am. B 12, 2229 (1995).

[29] Y. Yin, J. Li, X. Ren, K. Zhao, Y. Wu, E. Cunningham, and Z. Chang, High-efficiency optical parametric chirped-pulse amplifier in $\mathrm{BiB}_{3} \mathrm{O}_{6}$ for generation of $3 \mathrm{~mJ}$, two-cycle, carrierenvelope-phase-stable pulses at $1.7 \mu \mathrm{m}$, Opt. Lett. 41, 1142 (2016).

[30] Y. P. Deng, A. Schwarz, H. Fattahi, M. Ueffing, X. Gu, M. Ossiander, T. Metzger, V. Pervak, H. Ishizuki, T. Taira et al., Carrier-envelope-phase-stable, $1.2 \mathrm{~mJ}, 1.5$ cycle laser pulses at $2.1 \mu \mathrm{m}$, Opt. Lett. 37, 4973 (2012).

[31] N. Ishii, K. Kaneshima, T. Kanai, S. Watanabe, and J. Itatani, Generation of sub-two-cycle millijoule infrared pulses in an optical parametric chirped-pulse amplifier and their application to soft x-ray absorption spectroscopy with high-flux high harmonics, J. Opt. 20, 014003 (2018).

[32] C. Rolland and P. B. Corkum, Compression of high-power optical pulses, J. Opt. Soc. Am. B 5, 641 (1988).

[33] E. Mével, O. Tcherbakoff, F. Salin, and E. Constant, Extracavity compression technique for high-energy femtosecond pulses, J. Opt. Soc. Am. B 20, 105 (2003).

[34] V. Petrov, W. Rudolph, and B. Wilhelmi, Compression of highenergy femtosecond light pulses by self-phase modulation in bulk media, J. Mod. Opt. 36, 587 (1989).

[35] D. H. Reitze, A. M. Weiner, and D. E. Leaird, High-power femtosecond optical pulse compression by using spatial solitons, Opt. Lett. 16, 1409 (1991)

[36] V. Shumakova, P. Malevich, S. Ališauskas, A. Voronin, A. M. Zheltikov, D. Faccio, D. Kartashov, A. Baltuška, and A. Pugžlys, Multi-millijoule few-cycle mid-infrared pulses through nonlinear self-compression in bulk, Nat. Commun. 7, 12877 (2016).

[37] M. B. Schwab, A. Savert, O. Jackel, and J. Polz, Few-cycle optical probe-pulse for investigation of relativistic laser-plasma interactions, Appl. Phys. Lett. 103, 191118 (2013).

[38] D. J. Frantzeskakis, H. Leblond, and D. Mihalache, Nonlinear optics of intense few-cycle pulses: an overview of recent theoretical and experimental developments, Romanian J. Phys. 59, 767 (2014).

[39] A. Wirth, M. T. Hassan, I. Grguras, J. Gagnon, A. Moulet, T. T. Luu, S. Pabst, R. Santra, Z. A. Alahmed, A. M. Azzeer et al., Synthesized light transients, Science 334, 195 (2011). 
[40] H. Jacqmin, A. Jullien, B. Mercier, M. Hanna, F. Druon, D. Papadopoulos, and R. Lopez-Martens, Passive coherent combining of CEP-stable few-cycles pulses from a temporally divided hollow fiber compressor, Opt. Lett. 40, 709 (2015).

[41] P. Huang, S. B. Fang, Y. T. Gao, K. Zhao, X. Hou, and Z. Y. Wei, Simple method for simultaneous long-term stabilization of relative timing and carrier-envelope phase in waveform synthesis, Appl. Phys. Lett. 115, 031102 (2019).

[42] J. Rothhardt, S. Hädrich, A. Klenke, S. Demmler, A. Hoffmann, T. Gotschall, T. Eidam, M. Krebs, J. Limpert, and A. Tünnermann, $53 \mathrm{~W}$ average power few-cycle fiber laser system generating soft $\mathrm{x}$ rays up to the water window, Opt. Lett. 39, 5224 (2014).

[43] S. Hädrich, A. Klenke, J. Rothhardt, M. Krebs, A. Hoffmann, O. Pronin, V. Pervak, J. Limpert, and A. Tünnermann, High photon flux table-top coherent extreme-ultraviolet source, Nat. Photonics 8, 779 (2014).

[44] E. Goulielmakis, M. Schultze, M. Hofstetter, V. S. Yakovlev, J. Gagnon, M. Uiberacker, A. L. Aquita, E. M. Gullikson, D. T. Attwood, and R. Kienberger, Single-cycle nonlinear optics, Science 320, 1614 (2008).

[45] K. Zhao, Q. Zhang, M. Chini, Y. Wu, X. Wang, and Z. Chang, Tailoring a 67 attosecond pulse through advantageous phasemismatch, Opt. Lett. 37, 3891 (2012).

[46] P. B. Corkum, C. Rolland, and T. Srinivasan-Rao, Supercontinuum Generation in Gases, Phys. Rev. Lett. 57, 2268 (1986).

[47] C. P. Hauri, W. Kornelis, F. W. Helbing, A. Heinrich, A. Couairon, A. Mysyrowicz, J. Biegert, and U. Keller, Generation of intense, carrier-envelope phase-locked few-cycle laser pulses through filamentation, Appl. Phys. B 79, 673 (2004).

[48] P. Balla, A. Bin Wahid, I. Sytcevich, C. Guo, A.-L. Viotti, L. Silletti, A. Cartella, S. Alisauskas, H. Tavakol, U. GrosseWortmann et al., Postcompression of picosecond pulses into the few-cycle regime, Opt. Lett. 45, 2572 (2020).

[49] A. V. Mitrofanov, D. A. Sidorov-Biryukov, M. M. Nazarov, A. A. Voronin, M. V. Rozhko, A. D. Shutov, S. V. Ryabchuk, E. E. Serebryannikov, A. B. Fedotov, and A. M. Zheltikov, Ultraviolet-to-millimeter-band supercontinua driven by ultrashort mid-infrared laser pulses, Optica 7, 15 (2020).

[50] R. L. Fork, C. V. Shank, C. Hirlimann, R. Yen, and W. J. Tomlinson, Femtosecond white-light continuum pulses, Opt. Lett. 8, 1 (1983).

[51] R. W. Schoenlein, J. Y. Bigot, M. T. Portella, and C. V. Shank, Generation of blue-green $10 \mathrm{fs}$ pulses using an excimer pumped dye amplifier, Appl. Phys. Lett. 58, 801 (1991).

[52] A. Brodeur and S. L. Chin, Band-Gap Dependence of the Ultrafast White-Light Continuum, Phys. Rev. Lett. 80, 4406 (1998).

[53] A. Brodeur and S. L. Chin, Ultrafast white-light continuum generation and self-focusing in transparent condensed media, J. Opt. Soc. Am. B 16, 637 (1999).

[54] N. Krebs, I. Pugliesi, and E. Riedle, Pulse compression of ultrashort UV pulses by self-phase modulation in bulk material, Appl. Sci. 3, 153 (2013).

[55] C. H. Lu, Y. J. Tsou, H. Y. Chen, B. H. Chen, Y. C. Cheng, S. D. Yang, M. C. Chen, C. Hsu, and A. H. Kung, Generation of intense supercontinuum in condensed media, Optica 1, 400 (2014).

[56] H. Liang, P. Krogen, R. Grynko, O. Novak, C. L. Chang, G. J. Stein, D. Weerawarne, B. Shim, F. X. Kärtner, and
K. H. Hong, Three-octave-spanning supercontinuum generation and sub-two-cycle self-compression of mid-infrared filaments in dielectrics, Opt. Lett. 40, 1069 (2015).

[57] F. Silva, D. R. Austin, A. Thai, M. Baudisch, M. Hemmer, D. Faccio, A. Couairon, and J. Biegert, Multi-octave supercontinuum generation from mid-infrared filamentation in a bulk crystal, Nat. Commun. 3, 807 (2012).

[58] Y. Su, S. B. Fang, Y. T. Gao, K. Zhao, G. Q. Chang, and Z. Y. Wei, Efficient generation of UV-enhanced intense supercontinuum in solids: toward sub-cycle transient, Appl. Phys. Lett. 118, 261102 (2021).

[59] W. Liu, S. Petit, A. Becker, N. Aközbek, C. M. Bowden, and S. L. Chin, Intensity clamping of a femtosecond laser pulse in condensed matter, Opt. Commun. 202, 189 (2002).

[60] M. Bradler, P. Baum, and E. Riedle, Femtosecond continuum generation in bulk laser host materials with sub- $\mu \mathrm{J}$ pump pulses, Appl. Phys. B 97, 561 (2009).

[61] M. Hemmer, M. Baudisch, A. Thai, A. Couairon, and J. Biegert, Self-compression to sub-3-cycle duration of midinfrared optical pulses in dielectrics, Opt. Express 21, 28095 (2013).

[62] A. A. Lanin, A. A. Voronin, E. A. Stepanov, A. B. Fedotov, and A. M. Zheltikov, Multioctave, 3-18 $\mu \mathrm{m}$ sub-two-cycle supercontinua from self-compressing, self-focusing soliton transients in a solid, Opt. Lett. 40, 974 (2015).

[63] M. Centurion, M. A. Porter, P. G. Kevrekidis, and D. Psaltis, Nonlinearity Management in Optics: Experiment, Theory, and Simulation, Phys. Rev. Lett. 97, 033903 (2006).

[64] P. He, Y. Y. Liu, K. Zhao, H. Teng, X. K. He, H. Pei, H. D. Huang, S. Y. Zhong, Y. J. Jiang, S. B. Fang et al., Highefficiency supercontinuum generation in solid thin plates at 0.1 TW level, Opt. Lett. 42, 474 (2017).

[65] Y. Y. Liu, K. Zhao, P. He, H. D. Huang, H. Teng, and Z. Y. Wei, High-efficiency generation of $0.12 \mathrm{~mJ}, 8.6 \mathrm{fs}$ pulses at $400 \mathrm{~nm}$ based on spectral broadening in solid thin plates, Chin. Phys. Lett. 34, 074204 (2017).

[66] C. H. Lu, T. Witting, A. Husakou, M. J. J. Vrakking, A. H. Kung, and F. J. Furch, Sub-4 fs laser pulses at high average power and high repetition rate from an all-solid-state setup, Opt. Express 26, 8941 (2018).

[67] F. M. Lu, P. Y. Xia, Y. Matsumoto, T. Kanai, N. Ishii, and J. Itatani, Generation of sub-two-cycle CEP-stable optical pulses at $3.5 \mu \mathrm{m}$ from a KTA-based optical parametric amplifier with multiple-plate compression, Opt. Lett. 43, 2720 (2018).

[68] K. Zhao, S. Y. Xu, Y. J. Jiang, Y. T. Gao, Y. Y. Liu, P. He, H. Teng, J. F. Zhu, and Z. Y. Wei, Research progress on octave supercontinuum generation in solid medium [in Chinese], Acta Phys. Sin. 67, 124203 (2018).

[69] M. Canhota, R. Weigand, and H. M. Crespo, Simultaneous measurement of two ultrashort near-ultraviolet pulses produced by a multiplate continuum using dual self-diffraction dispersion-scan, Opt. Lett. 44, 1015 (2019).

[70] C. L. Tsai, Y. H. Tseng, A. Y. Liang, M. W. Lin, S. D. Yang, and M. C. Chen, Nonlinear compression of intense optical pulses at $1.55 \mu \mathrm{m}$ by multiple plate continuum generation, J. Lightwave Technol. 37, 5100 (2019).

[71] H. B. Cao, R. S. Nagymihaly, N. Khodakovskiy, V. Pajer, J. Bohus, R. Lopez-Martens, A. Borzsonyi, and M. Kalashnikov, Sub-7 fs radially-polarized pulses by post-compression in thin fused silica plates, Opt. Express 29, 5915 (2021). 
[72] B. Shao, Y. Li, Y. Peng, W. Li, J. Qian, Y. X. Leng, and R. X. Li, $1.9 \mu \mathrm{m}$ few-cycle pulses based on multi-thin-plate spectral broadening and nonlinear self-compression, IEEE Photon. J. 13, 3300108 (2021).

[73] R. R. Tamming, C. Y. Lin, J. M. Hodgkiss, S. D. Yang, K. Chen, and C. H. Lu, Single 3.3 fs multiple plate compression light source in ultrafast transient absorption spectroscopy, Sci. Rep. 11, 12847 (2021).

[74] S. Kedenburg, T. Steinle, F. Mörz, A. Steinmann, and H. Giessen, High-power mid-infrared high repetition-rate supercontinuum source based on a chalcogenide step-index fiber, Opt. Lett. 40, 2668 (2015).

[75] R. L. Fork, C. H. B. Cruz, P. C. Becker, and C. V. Shank, Compression of optical pulses to six femtoseconds by using cubic phase compensation, Opt. Lett. 12, 483 (1987).

[76] A. Baltuska, Z. Y. Wei, M. S. Pshenichnikov, D. A. Wiersma, and R. Szipöcs, All-solid-state cavity-dumped sub-5-fs laser, Appl. Phys. B 65, 175 (1997).

[77] M. Nisoli, S. D. Silvestri, and O. Svelto, Generation of high energy 10 fs pulses by a new pulse compression technique, Appl. Phys. Lett. 68, 2793 (1996).

[78] W. Zhang, H. Teng, C. X. Yun, X. Zhong, X. Hou, and Z. Y. Wei, Generation of sub-2 cycle optical pulses with a differentially pumped hollow fiber, Chin. Phys. Lett. 27, 054211 (2010).

[79] V. Cardin, N. Thiré, S. Beaulieu, V. Wanie, F. Légaré, and B. E. Schmidt, 0.42 TW 2-cycle pulses at $1.8 \mu \mathrm{m}$ via hollow-core fiber compression, Appl. Phys. Lett. 107, 181101 (2015).

[80] A. Suda, M. Hatayama, K. Nagasaka, and K. Midorikawa, Generation of sub-10-fs, 5-mJ-optical pulses using a hollow fiber with a pressure gradient, Appl. Phys. Lett. 86, 111116 (2005).

[81] G. Steinmeyer and G. Stibenz, Generation of sub-4-fs pulses via compression of a white-light continuum using only chirped mirrors, Appl. Phys. B 82, 175 (2006).

[82] S. Bohman, A. Suda, T. Kanai, S. Yamaguchi, and K. Midorikawa, Generation of $5.0 \mathrm{fs}, 5.0 \mathrm{~mJ}$ pulses at $1 \mathrm{kHz}$ using hollow-fiber pulse compression, Opt. Lett. 35, 1887 (2010).

[83] C. F. Dutin, A. Dubrouil, S. Petit, E. Mével, E. Constant, and D. Descamps, Post-compression of high-energy femtosecond pulses using gas ionization, Opt. Lett. 35, 253 (2010).

[84] F. Silva, B. Alonso, W. Holgado, R. Romero, J. S. Román, E. C. Jarque, H. Koop, V. Pervak, H. Crespo, and Í. J. Sola, Strategies for achieving intense single-cycle pulses with inline post-compression setups, Opt. Lett. 43, 337 (2018).

[85] T. Nagy, S. Hädrich, P. Simon, A. Blumenstein, N. Walther, R. Klas, J. Buldt, H. Stark, S. Breitkopf, P. Jójárt et al., Generation of three-cycle multi-millijoule laser pulses at $318 \mathrm{~W}$ average power, Optica 6, 1423 (2019).

[86] T. Nagy, M. Kretschmar, M. J. J. Vrakking, and A. Rouzée, Generation of above-terawatt 1.5 -cycle visible pulses at $1 \mathrm{kHz}$ by post-compression in a hollow fiber, Opt. Lett. 45, 3313 (2020).

[87] G. Fan, P. A. Carpeggiani, Z. Tao, G. Coccia, R. Safaei, E. Kaksis, A. Pugzlys, F. Légaré, B. E. Schmidt, and A. Baltuška, $70 \mathrm{~mJ}$ nonlinear compression and scaling route for an $\mathrm{Yb}$ amplifier using large-core hollow fibers, Opt. Lett. 46, 896 (2021).
[88] F. Belli, A. Abdolvand, W. Chang, J. C. Travers, and P. Russell, Vacuum-ultraviolet to infrared supercontinuum in hydrogenfilled photonic crystal fiber, Optica 2, 292 (2015).

[89] J. Huang, M. Pang, X. Jiang, F. Köttig, D. Schade, W. He, M. Butryn, and P. S. J. Russell, Sub-two-cycle octave-spanning mid-infrared fiber laser, Optica 7, 574 (2020).

[90] F. Leo, S. P. Gorza, S. Coen, B. Kuyken, and G. Roelkens, Coherent supercontinuum generation in a silicon photonic wire in the telecommunication wavelength range, Opt. Lett. 40, 123 (2014).

[91] H. Zhao, B. Kuyken, S. Clemmen, F. O. Leo, A. Subramanian, A. Dhakal, P. Helin, S. Severi, E. Brainis, and G. Roelkens, Visible-to-near-infrared octave spanning supercontinuum generation in a silicon nitride waveguide, Opt. Lett. 40, 2177 (2015).

[92] M. Rutkauskas, A. Srivastava, and D. T. Reid, Supercontinuum generation in orientation-patterned gallium phosphide, Optica 7, 172 (2020).

[93] D. Wang, D. X. Li, X. W. Chen, Y. X. Leng, and Z. Z. Xu, Few-cycle pulse compression through cascade of bulk media and hollow-core fiber, Laser Phys. 20, 1410 (2010).

[94] S. Mironov, P. Lassonde, J. C. Kieffer, E. Khazanov, and G. Mourou, Spatially-uniform temporal recompression of intense femtosecond optical pulses, Eur. Phys. J. Spec. Top. 223, 1175 (2014).

[95] R. Budriūnas, D. Kučinskas, and A. Varanavičius, Highenergy continuum generation in an array of thin plates pumped by tunable femtosecond IR pulses, Appl. Phys. B 123, 212 (2017).

[96] T. K. Gustafson, J. P. Taran, H. A. Haus, J. R. Lifsitz, and P. L. Kelley, Self-modulation, self-steepening, and spectral development of light in small-scale trapped filaments, Phys. Rev. 177, 306 (1969).

[97] J. E. Rothenberg, Space-time focusing: Breakdown of the slowly varying envelope approximation in the self-focusing of femtosecond pulses, Opt. Lett. 17, 1340 (1992).

[98] A. L. Gaeta, Catastrophic Collapse of Ultrashort Pulses, Phys. Rev. Lett. 84, 3582 (2000).

[99] L. Sudrie, A. Couairon, M. Franco, B. Lamouroux, B. Prade, S. Tzortzakis, and A. Mysyrowicz, Femtosecond LaserInduced Damage and Filamentary Propagation in Fused Silica, Phys. Rev. Lett. 89, 186601 (2002).

[100] N. T. Nguyen, A. Saliminia, W. Liu, S. L. Chin, and R. Vallée, Optical breakdown versus filamentation in fused silica by use of femtosecond infrared laser pulses, Opt. Lett. 28, 1591 (2003).

[101] A. Couairon, L. Sudrie, M. Franco, B. Prade, and A. Mysyrowicz, Filamentation and damage in fused silica induced by tightly focused femtosecond laser pulses, Phys. Rev. B 71, 125435 (2005)

[102] A. Couairon and A. Mysyrowicz, Femtosecond filamentation in transparent media, Phys. Rep. 441, 47 (2007).

[103] S. N. Vlasov, E. V. Koposova, and V. E. Yashin, Spectral broadening and compression of high-intensity laser pulses in quasi-periodic systems with Kerr nonlinearity, Quantum Electron. 42, 989 (2012)

[104] A. A. Voronin, A. M. Zheltikov, T. Ditmire, B. Rus, and G. Korn, Subexawatt few-cycle lightwave generation via multipetawatt pulse compression, Opt. Commun. 291, 299 (2013). 
[105] J. E. Beetar, S. Gholam-Mirzaei, and M. Chini, Spectral broadening and pulse compression of a $400 \mu \mathrm{J}, 20 \mathrm{~W}$ Yb:KGW laser using a multi-plate medium, Appl. Phys. Lett. 112, 051102 (2018).

[106] S. I. Hwang, S. B. Park, J. Mun, W. Cho, C. H. Nam, and K. T. Kim, Generation of a single-cycle pulse using a twostage compressor and its temporal characterization using a tunnelling ionization method, Sci. Rep. 9, 1613 (2019).

[107] N. Ishii, P. Xia, T. Kanai, and J. Itatani, Optical parametric amplification of carrier-envelope phase-stabilized mid-infrared pulses generated by intra-pulse difference frequency generation, Opt. Express 27, 11447 (2019).

[108] C. H. Lu, W. H. Wu, S. H. Kuo, J. Y. Guo, M. C. Chen, S. D. Yang, and A. Kung, Greater than 50 times compression of $1030 \mathrm{~nm} \mathrm{Yb}: \mathrm{KGW}$ laser pulses to single-cycle duration, Opt. Express 27, 15638 (2019).

[109] S. Y. Mironov, S. Fourmaux, P. Lassonde, V. N. Ginzburg, and G. Mourou, Thin plate compression of a sub-petawatt Ti:Sa laser pulses, Appl. Phys. Lett. 116, 241101 (2020).

[110] M. Seo, K. Tsendsuren, S. Mitra, M. Kling, and D. Kim, High-contrast, intense single-cycle pulses from an all thinsolid-plate setup, Opt. Lett. 45, 367 (2019).

[111] V. N. Ginzburg, I. V. Yakovlev, A. S. Zuev, A. P. Korobeynikova, A. A. Kochetkov, A. A. Kuzmin, S. Y. Mironov, A. A. Shaykin, I. A. Shaikin, and E. A. Khazanov, Two-stage nonlinear compression of high-power femtosecond laser pulses, Quantum Electron. 50, 331 (2020).

[112] M. Stanfield, N. F. Beier, S. Hakimi, H. Allison, D. Farinella, A. E. Hussein, T. Tajima, and F. Dollar, Millijoule few cycle pulses from staged compression for strong and high field science, Opt. Express 29, 9123 (2021).

[113] D. M. Farinella, J. Wheeler, A. E. Hussein, J. Nees, M. Stanfield, N. Beier, Y. Ma, G. Cojocaru, R. Ungureanu, M. Pittman et al., Focusability of laser pulses at petawatt transport intensities in thin-film compression, J. Opt. Soc. Am. B 36, A28 (2019).

[114] O. G. Kosareva, V. P. Kandidov, A. Brodeur, C. Y. Chien, and S. L. Chin, Conical emission from laser-plasma interactions in the filamentation of powerful ultrashort laser pulses in air, Opt. Lett. 22, 1332 (1997).

[115] F. Théberge, M. Châteauneuf, V. Ross, P. Mathieu, and J. Dubois, Ultrabroadband conical emission generated from the ultraviolet up to the far-infrared during the optical filamentation in air, Opt. Lett. 33, 2515 (2008).

[116] S. Trillo, C. Conti, P. D. Trapani, O. Jedrkiewicz, J. Trull, G. Valiulis, and G. Bellanca, Colored conical emission by means of second-harmonic generation, Opt. Lett. 27, 1451 (2002).

[117] D. Faccio, M. A. Porras, A. Dubietis, F. Bragheri, A. Couairon, and P. DiTrapani, Conical Emission, Pulse Splitting and X-Wave Parametric Amplification in Nonlinear Dynamics of Ultrashort Light Pulses, Phys. Rev. Lett. 96, 193901 (2006).

[118] F. Bragheri, D. Faccio, A. Couairon, A. Matijosius, G. Tamosauskas, A. Varanavicius, V. Degiorgio, A. Piskarskas, and P. DiTrapani, Conical-emission and shock-front dynamics in femtosecond laser-pulse filamentation, Phys. Rev. A 76, 025801 (2007).

[119] Y. T. Gao, Y. B. Su, S. Y. Xu, X. X. Zhu, K. Zhao, S. B. Fang, J. F. Zhu, and Z. Y. Wei, Generation of annular femtosecond few-cycle pulses by self-compression and spatial filtering in solid thin plates, Opt. Express 29, 29789 (2021).
[120] N. Bloembergen, The influence of electron plasma formation on superbroadening in light filaments, Opt. Commun. 8, 285 (1973).

[121] M. D. Perry, T. Ditmire, and B. C. Stuart, Self-phase modulation in chirped-pulse amplification, Opt. Lett. 19, 2149 (1994).

[122] G. Genty, L. Salmela, J. M. Dudley, D. Brunner, and S. K. Turitsyn, Machine learning and applications in ultrafast photonics, Nat. Photonics 15, 91 (2020).

[123] S. Y. Xu, X. X. Zhu, J. Wang, Y. F. Li, Y. T. Gao, K. Zhao, J. F. Zhu, D. C. Zhang, Y. L. Chen, and Z. Y. Wei, Convolutional neural network for transient grating frequency-resolved optical gating trace retrieval and its algorithm optimization, Chin. Phys. B 30, 048402 (2021).

[124] K. M. Hornik, M. Stinchcomb, and H. White, Multilayer feedforward networks are universal approximator, Neural Netw. 2, 359 (1989).

[125] L. Berge, S. Skupin, R. Nuter, J. Kasparian, and J. P. Wolf, Ultrashort filaments of light in weakly-ionized, opticallytransparent media, Rep. Prog. Phys. 70, 1633 (2006).

[126] G. Genty, A. T. Friberg, and J. Turunen, Coherence of supercontinuum light, Prog. Opt. 61, 71 (2016).

[127] J. M. Dudley and S. Coen, Coherence properties of supercontinuum spectra generated in photonic crystal and tapered optical fibers, Opt. Lett. 27, 1180 (2002).

[128] V. Sitzmann, J. N. P. Martel, A. W. Bergman, D. B. Lindell, and G.Wetzstein, Implicit neural representations with periodic activation functions, in Advances in Neural Information Processing Systems, edited by H. Larochelle, M. Ranzato, R. Hadsell, M. F. Balcan, and H. Lin (Curran Associates, Inc., 2020), Vol. 33, pp. 74-62.

[129] D. Kingma and J. Ba, Adam: A Method for Stochastic Optimization, the 3rd International Conference for Learning Representations (San Diego, 2015).

[130] S. Qin, Z. H. Wang, S. S. Yang, Z. W. Shen, Q. L. Dong, and Z. Y. Wei, Spectral-phase-modulated cross-polarized wave for chirped pulse amplifier with high contrast ratio, Chin. Phys. Lett. 34, 024205 (2017).

[131] L. Salmela, C. Lapre, J. M. Dudley, and G. Genty, Machine learning analysis of rogue solitons in supercontinuum generation, Sci. Rep. 10, 9596 (2020).

[132] N. Raabe, T. Feng, T. Witting, A. Demircan, C. Brée, and G. Steinmeyer, Role of Intrapulse Coherence in Carrier-Envelope Phase Stabilization, Phys. Rev. Lett. 119, 123901 (2017).

[133] Y. T. Gao, Y. Y. Liu, P. Huang, S. Y. Xu, Y. J. Jiang, J. Wang, K. Zhao, S. B. Fang, and Z. Y. Wei, in The 9th International Symposium on Ultrafast Phenomena and Terahertz Waves (Optical Society of America, Changsha, 2018), p. WI38.

[134] L. Shah, Z. Liu, I. Hartl, G. Imeshev, G. Cho, and M. Fermann, High energy femtosecond $\mathrm{Yb}$ cubicon fiber amplifier, Opt. Express 13, 4717 (2005)

[135] S. A. Zhou, L. Kuznetsova, A. Chong, and F. W. Wise, Compensation of nonlinear phase shifts with third-order dispersion in short-pulse fiber amplifiers, Opt. Express 13, 4869 (2005).

[136] K. Fritsch, M. Poetzlberger, V. Pervak, J. Brons, and O. Pronin, All-solid-state multipass spectral broadening to sub-20 fs, Opt. Lett. 43, 4643 (2018).

Correction: The article identification number in Ref. [5] was incorrect and has been fixed, enabling access to the intended article. 